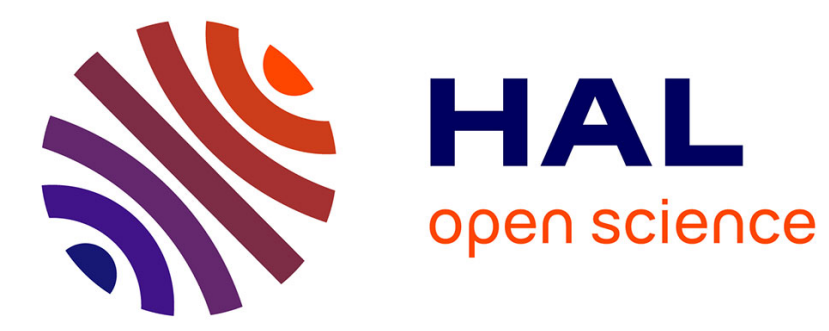

\title{
A Unified View of Exact Continuous Penalties for 12-10 Minimization
}

\author{
Emmanuel Soubies, Laure Blanc-Féraud, Gilles Aubert
}

\section{To cite this version:}

Emmanuel Soubies, Laure Blanc-Féraud, Gilles Aubert. A Unified View of Exact Continuous Penalties for 12-10 Minimization. SIAM Journal on Optimization, 2017, 27 (3), 10.1137/16M1059333 . hal$01267701 \mathrm{v} 2$

\section{HAL Id: hal-01267701 \\ https://hal.inria.fr/hal-01267701v2}

Submitted on 11 Jul 2017

HAL is a multi-disciplinary open access archive for the deposit and dissemination of scientific research documents, whether they are published or not. The documents may come from teaching and research institutions in France or abroad, or from public or private research centers.
L'archive ouverte pluridisciplinaire HAL, est destinée au dépôt et à la diffusion de documents scientifiques de niveau recherche, publiés ou non, émanant des établissements d'enseignement et de recherche français ou étrangers, des laboratoires publics ou privés. 


\title{
A Unified View of Exact Continuous Penalties for $\ell_{2}-\ell_{0}$ Minimization.
}

\author{
Emmanuel Soubies ${ }^{\dagger}$ \\ Laure Blanc-Féraud ${ }^{\dagger}$ \\ Gilles Aubert ${ }^{\ddagger}$
}

July 11, 2017

\begin{abstract}
Numerous nonconvex continuous penalties have been proposed to approach the $\ell_{0}$ pseudonorm for optimization purpose. Apart from the theoretical results for convex $\ell_{1}$ relaxation under restrictive hypotheses, only few works have been devoted to analyze the consistency, in terms of minimizers, between the $\ell_{0}$-regularized least square functional and relaxed ones using continuous approximations. In this context, two questions are of fundamental importance: does relaxed functionals preserve global minimizers of the initial one? Does these approximations introduce unwanted new (local) minimizers? In this paper we answer these questions by deriving necessary and sufficient conditions on such $\ell_{0}$ continuous approximations in order that each (local and global) minimizer of the underlying relaxation is also a minimizer of the $\ell_{2}-\ell_{0}$ functional and that all the global minimizers of the initial functional are preserved. Hence, a general class of penalties is provided giving a unified view of exact continuous approximations of the $\ell_{0}$-norm within the $\ell_{2}$ $\ell_{0}$ minimization framework. As the inferior limit of this class of penalties, we get the recently proposed CEL0 penalty. Finally, state of the art penalties, such as MCP, SCAD or Capped- $\ell_{1}$, are analyzed according to the proposed class of exact continuous penalties.
\end{abstract}

Key words $\ell_{0}$-regularized least squares, exact reformulation, exact $\ell_{0}$ penalties, sparse modeling, underdeterminated linear system, global minimizers, local minimizers, minimizers equivalence

\section{Introduction}

In this paper, we are concerned with the following $\ell_{0}$ regularized least squares problem,

$$
\hat{\mathrm{x}} \in \arg \min _{\mathrm{x} \in \mathbb{R}^{N}} \mathrm{G}_{\ell_{0}}(\mathrm{x}):=\frac{1}{2}\|A \mathrm{x}-d\|_{2}^{2}+\lambda\|\mathrm{x}\|_{0},
$$

where $A \in \mathbb{R}^{M \times N}$ (usually $\left.M \ll N\right), d \in \mathbb{R}^{M}$ and $\|\cdot\|_{0}$ denotes the so-called " $\ell_{0}$-norm" defined by,

$$
\|\mathrm{x}\|_{0}=\sharp\left\{\mathrm{x}_{i}, i=1, \ldots, N: \mathrm{x}_{i} \neq 0\right\},
$$

with $\sharp$ denoting the cardinality. In other words, the $\ell_{0}$-norm counts the nonzero entries of $x$. Finally, $\lambda>0$ is a hyperparameter characterizing a trade-off between data fidelity and sparsity. This problem finds a wide range of applications in signal/image processing, learning and coding areas among

\footnotetext{
${ }^{\dagger}$ Université de Nice Sophia Antipolis, CNRS, Laboratoire I3S UMR 7271, 06903 Sophia Antipolis, France (soubies@i3s.unice.fr, blancf@i3s.unice.fr). Authors belong to the Morpheme team (UNS/CNRS/INRIA).

${ }^{\ddagger}$ Université de Nice Sophia Antipolis, CNRS, Laboratoire J.A. Dieudonné UMR 7351, 06100 Nice, France (gilles.aubert@unice.fr)
} 
many others. Constrained versions of (1) have also been extensively used and studied over the last decades. However, due to their nonconvexity, $\ell_{0}$-constrained and $\ell_{0}$-regularized $(1)$ problems are not (completely) equivalent [21, 32]. Moreover, a combinatorial search to tackle such problems is known to be NP-Hard [19] and considerable efforts have been done and continue to be done in order to design efficient methods/algorithms to find (approximate) solutions of (1) (or its constrained forms).

Convex relaxation, where the $\ell_{0}$-norm is replaced by the $\ell_{1}$-norm, became very popular after the theoretical results of Donoho [10] and, Candès, Romberg and Tao [5] showing that under suitable conditions on $A$ (e.g. RIP criteria, incoherence assumption...), sufficiently sparse signals can be exactly recovered by $\ell_{1}$ minimization. However, these conditions are quite restrictive for many practical applications. Other common approaches to deal with such problems are greedy methods such as matching pursuit (MP) [18], orthogonal matching pursuit (OMP) [23] or single best replacement (SBR) [27]. Similar recovery guarantiees as for the $\ell_{1}$ relaxation have been established for OMP in [29]. Besides, continuous nonconvex approximations have received considerable attention and numerous penalties have been proposed to approach the $\ell_{0}$-norm. Among the variety of $\ell_{0}$-like continuous penalties, one can find the nonnegative garrote [4], log-sum penalty [6], capped- $\ell_{1}[33], \ell_{p}$-norms $(0<p<1)[12]$, ratio $\ell_{1} / \ell_{2}$ [25], smoothly clipped absolute deviation (SCAD) [11], minimax concave penalty (MCP) [31] or the reverse huber penalty [24]. Note that the two last penalties can also be derived using perspective relaxation [9]. Such penalties have been shown to better promote sparsity than $\ell_{1}$-norm and to avoid (reduce) the bias introduced by the $\ell_{1}$ penalty on large coefficients [34, 11]. Although Fan and Li [11] designed conditions that a "good" penalty function must satisfy (unbiasedness, continuity in data, sparsity) and that Zhang [30] proposed the notion of sparse convexity to compare penalties, the choice of one continuous nonconvex penalty rather than another remains unclear. Moreover, only few works have been dedicated to analyze the links between the underlying relaxed problems and the original one (involving the $\ell_{0}$-norm).

A class of smooth nonconvex penalties have been proposed to approach the $\ell_{0}$-norm in [7] and asymptotic connections with the $\ell_{0}$ penalized criteria, in terms of global minimizers, have been shown. In [13], the authors showed the equivalence between $\ell_{0^{-}}$and $\ell_{p}$-norm minimization under linear equalities or inequalities. More precisely, after reformulating these two problems as the minimization of a non-convex cost function over a bounded feasible region, which is the same for both problems, the main result of that paper states the existence of a vertex of this set which is solution of both $\ell_{0^{-}}$and $\ell_{p^{-}}$minimization problems for some $p \leq 1$. Mixed-integer programming (MIP) reformulations of $\ell_{0^{-}}$ norm based criteria have recently been proposed in [3] allowing exact optimization through branch and bound based algorithms together with cutting plane methods. However, due to computing time issues, such methods are restricted to moderate-size problems involving hundreds of variables. By considering problem (1) with a finite DC (Difference of Convex functions) data term and an additional constraint $\mathrm{x} \in K \subset \mathbb{R}^{N}$, where $K$ is a polyhedral convex set, the authors in [16] have proposed a family of continuous DC approximations of the $\ell_{0}$-norm and showed links between the approximated and original problems. They proved that any minimizer of the approximated problem is in a $\varepsilon$-neighbourhood of a minimizer of the initial problem. Moreover, when the data term functional is concave and bounded below on $K$, they showed that optimal solutions of the approximated problem are included in the ones of the initial problem. Then, using an exact continuous reformulation of the problem as a DC program, a stronger result is showed for the Capped- $\ell_{1}$ penalty (see also [17]), for which global solutions of the approximation exactly coincides with the ones of the initial functional. However these two last results are limited to global solutions and are not available for local minimizers. At the same time, we have proposed the continuous exact $\ell_{0}$ (CEL0) penalty [26] leading to stronger properties (in the case of problem (1)) since we proved that the resulting tight continuous relaxation, $\mathrm{G}_{\mathrm{CEL} \text {. }}$, preserves global minimizers of $\mathrm{G}_{\ell_{0}}$ while some local minimizers are removed (the others remain unchanged). 
Following the recent works [16] and [26], it seems of interest to give a unified view of $\ell_{0}$-norm continuous approximations leading to nonconvex equivalent (in terms of minimizers) continuous relaxations of $\mathrm{G}_{\ell_{0}}$.

Contributions and outline In this work, we consider continuous approximations of the $\ell_{0}$-norm of the form

$$
\Phi(\mathrm{x})=\sum_{i=1}^{N} \phi_{i}\left(\mathrm{x}_{i}\right),
$$

where $\phi_{i}$ are continuous $1 \mathrm{D}$ penalties approximating the weighted " $0-1$ " function ${ }^{1} \lambda|\cdot|_{0}$, leading to continuous relaxations of $\mathrm{G}_{\ell_{0}}$ in (1) defined by

$$
\tilde{\mathrm{G}}(\mathrm{x})=\frac{1}{2}\|A \mathrm{x}-d\|_{2}^{2}+\Phi(\mathrm{x})
$$

Given $A \in \mathbb{R}^{M \times N}$, the present paper is devoted to the determination of necessary and sufficient conditions on $\phi_{i}$ (which may depend on the elements of $A$ but do not require any assumption on $A$ ) ensuring the two following properties for all $d \in \mathbb{R}^{M}$ :

$$
\begin{gathered}
\arg \min _{\mathrm{x} \in \mathbb{R}^{N}} \tilde{\mathrm{G}}(\mathrm{x})=\arg \min _{\mathrm{x} \in \mathbb{R}^{N}} \mathrm{G}_{\ell_{0}}(\mathrm{x}), \\
\hat{\mathrm{x}} \text { (local) minimizer of } \tilde{\mathrm{G}} \Longrightarrow \hat{\mathrm{x}} \text { (local) minimizer of } \mathrm{G}_{\ell_{0}} .
\end{gathered}
$$

In other words, we are concerned with the design of a class of continuous relaxations of $\mathrm{G}_{\ell_{0}}$, preserving all its global minimizers, and for which any local minimal point is also a local minimizer of the initial functional. From (P2), G can potentially eliminate local (not global) minimizers of $\mathrm{G}_{\ell_{0}}$ which is an interesting property for such highly nonconvex functional. Note that the approximating functions $\phi_{i}$ are depending on $\lambda$, may depend on $A$ but must not depend on $d$. Indeed, the approximation may depend on the given problem (which is defined by the matrix $A$ ) but not on the data so that the same relaxation can be used for all acquired data.

In Section 2, we consider the one dimensional case and prove five necessary and sufficient conditions to have (P1) and (P2). More precisely three conditions on the $1 \mathrm{D}$ continuous penalties are shown to be necessary and sufficient to have (P1) while two supplementary conditions are required to also have (P2). Then, Section 3 extends this result to the case where the matrix $A$, in the quadratic data fidelity term, has nonzero orthogonal columns. A further extension to any $A \in \mathbb{R}^{M \times N}$ is proposed in Section 4. This section provides the main contribution of this work where we show that, without any assumption on $A \in \mathbb{R}^{M \times N}$, the designed conditions are necessary and sufficient to have (P1) and (P2). We thereby highlight a class of continuous nonsmooth nonconvex penalties approximating the $\ell_{0}$-norm and leading to continuous relaxations of $\mathrm{G}_{\ell_{0}}$ for which properties (P1) and (P2) hold. It is worth noting that the CEL0 penalty proposed in [26] is the inferior limit of the obtained class of penalties. Finally, Section 5 analyses, within this context of exact continuous relaxation of $\mathrm{G}_{\ell_{0}}$, previously proposed " $\ell_{0}$-like" penalties such as MCP, SCAD or Capped- $\ell_{1}$. Using the general study conducted in Section 4, conditions on the parameters of these state of the art penalties are established in order to make $(\mathrm{P} 1)$ and $(\mathrm{P} 2)$ valid for the underlying relaxation $\tilde{\mathrm{G}}$.

\footnotetext{
${ }^{1}|\cdot|_{0}$ is defined by $|u|_{0}=1$ for $u \neq 0$ and $|u|_{0}=0$ for $u=0$.
} 
Notations and definitions We use the same notations as in [20, 26]:

- $\mathbb{I}_{N}=\{1, \ldots, N\}$,

- $a_{i} \in \mathbb{R}^{M}$, the $i$ th column of $A \in \mathbb{R}^{M \times N}$. We assume that $a_{i} \neq 0_{\mathbb{R}^{M}}, \forall i \in \mathbb{I}_{N}$,

- $\|\cdot\|=\|\cdot\|_{2}$ the $\ell_{2}$-norm. Otherwise we will precise the norm with a subscript,

- $e_{i} \in \mathbb{R}^{N}$, the unitary vector of the standard basis of $\mathbb{R}^{N}$,

- $\mathrm{x}^{(i)}=\left(\mathrm{x}_{1}, \ldots, \mathrm{x}_{i-1}, 0, \mathrm{x}_{i+1}, \ldots, \mathrm{x}_{N}\right) \in \mathbb{R}^{N}$,

- $A_{\omega}=\left(a_{\omega[1]}, \ldots, a_{\omega[\sharp \omega]}\right) \in R^{M \times \sharp \omega}$ for $\omega \subseteq \mathbb{I}_{N}$, the restriction of $A \in \mathbb{R}^{M \times N}$ to the columns indexed by the elements of $\omega \subseteq \mathbb{I}_{N}$,

- $\mathrm{x}_{\omega}=\left(\mathrm{x}_{\omega[1]}, \ldots, \mathrm{x}_{\omega[\sharp \omega]}\right) \in \mathbb{R}^{\sharp \omega}$ for $\omega \subseteq \mathbb{I}_{N}$, the restriction of $\mathrm{x} \in \mathbb{R}^{N}$ to the entries indexed by the elements of $\omega \subseteq \mathbb{I}_{N}$,

- $\sigma(\mathrm{x})=\left\{i \in \mathbb{I}_{N} ; \mathrm{x}_{i} \neq 0\right\} \subseteq \mathbb{I}_{N}$, the support of $\mathrm{x} \in \mathbb{R}^{N}$.

We recall the CEL0 penalty [26] which will be used in what follows:

$$
\Phi_{\text {CELo }}(\mathrm{x})=\sum_{i \in \mathbb{I}_{N}} \phi_{\text {CELo }}\left(\left\|a_{i}\right\|, \lambda ; \mathrm{x}_{i}\right)
$$

where for $a \in \mathbb{R}_{+}^{\star}, \lambda \in \mathbb{R}_{+}$and $u \in \mathbb{R}$,

$$
\phi_{\text {CELo }}(a, \lambda ; u)=\lambda-\frac{a^{2}}{2}\left(|u|-\frac{\sqrt{2 \lambda}}{a}\right)^{2} \mathbb{1}_{\left\{|u| \leq \frac{\sqrt{2 \lambda}}{a}\right\}} .
$$

Note that the CEL0 penalty is the same as MCP with a specific choice of parameters and a reparametrization. This specific choice of parameters leads to an exact continuous relaxation of $\mathrm{G}_{\ell_{0}}$ as shown in [26]. In the remaining of the paper we will refer to this specific MCP as CEL0 penalty.

\section{One dimensional analysis}

Let us consider the one dimensional problem,

$$
\hat{u} \in \arg \min _{u \in \mathbb{R}} g_{0}(u):=\frac{1}{2}(a u-d)^{2}+\lambda|u|_{0},
$$

where $a>0$ and $d \in \mathbb{R}$. Then, we are interested in a continuous approximation of $\lambda|\cdot|_{0}$, denoted $\phi$, leading to the following continuous relaxation of $g_{0}$ :

$$
\tilde{\mathrm{g}}(u):=\frac{1}{2}(a u-d)^{2}+\phi(u) .
$$

The goal is then to design necessary and sufficient conditions on $\phi$ such that $\tilde{\mathrm{g}}$ satisfies properties (P1) and (P2). In order to avoid penalties $\phi$ defined up to an additive constant, we introduce the following supplementary condition:

$$
\min _{u \in \mathbb{R}} \mathrm{g}_{0}(u)=\min _{u \in \mathbb{R}} \tilde{g}(u) .
$$

Then, we consider functions $\phi$ defined independently of $d$ (i.e. conditions derived on $\phi$ must not depend on $d$ ) to be able to extend the 1D case conditions to the ND case and so that the continuous approximation can be applied whatever the observations for a given problem (i.e. a given $a$ ). Moreover, throughout the paper we shall consider the two following assumptions on $\phi$. 
Assumption 1. $\phi$ is twice continuously differentiable $\left(C^{2}\right)$ on $\mathbb{R}$ except for finitely many points of $\mathbb{R}$, denoted by $B$. Moreover we assume that, for $v \in B$, the limits

$$
\lim _{\substack{u \rightarrow v \\ u<v}} \phi^{\prime}(u) \text { and } \lim _{\substack{u \rightarrow v \\ u>v}} \phi^{\prime}(u)
$$

exist, are finite, and are different (i.e. $\phi$ is not differentiable for points in B).

Assumption 2. $\phi$ is locally Lipschitz that is, $\forall u \in \mathbb{R}$,

$$
\left.\exists \varepsilon>0, \forall\left(v, v^{\prime}\right) \in\right] u-\varepsilon, u+\varepsilon\left[^{2},\left|\phi(v)-\phi\left(v^{\prime}\right)\right| \leq K_{u}\left|v-v^{\prime}\right|,\right.
$$

for some nonnegative scalar $K_{u}$.

The following proposition characterizes the global minimizer(s) of $\mathrm{g}_{0}$.

Proposition 3 (minimizers of $\mathrm{g}_{0}$ ). Let $a>0, \lambda>0$ and $d \in \mathbb{R}$. Then, a global minimizer, $u^{\star} \in \mathbb{R}$, of $\mathrm{g}_{0}$ verifies

$$
u^{\star}= \begin{cases}0 & \text { if }|d|<\sqrt{2 \lambda} \\ 0 \text { or } \frac{d}{a} & \text { if }|d|=\sqrt{2 \lambda} \\ \frac{d}{a} & \text { if }|d|>\sqrt{2 \lambda}\end{cases}
$$

Proof. It is clear that $\mathrm{g}_{0}$ has always two (local) minimizers $u_{1}=0$ and $u_{2}=\frac{d}{a}$. Then, noticing that

$$
\mathrm{g}_{0}(0)=\frac{d^{2}}{2} \text { and } \mathrm{g}_{0}\left(\frac{d}{a}\right)=\lambda,
$$

completes the proof.

In order to make $\tilde{g}$ preserving global minimizers of $g_{0}$, we first need to give a characterization of the critical points of $\tilde{\mathrm{g}}$ (i.e. points $\hat{u} \in \mathbb{R}$ such that $0 \in \partial \tilde{\mathrm{g}}(\hat{u})$ where $\partial \tilde{\mathrm{g}}$ denotes the generalized gradient of $\tilde{\mathrm{g}}[8]$ ) and ensure that global minimizers of $\mathrm{g}_{0}$ are critical points of $\tilde{\mathrm{g}}$. We first recall the definition of the Clarke generalized derivative of $\phi$ at $u \in \mathbb{R}$ in the direction $w \in \mathbb{R}$ :

$$
\phi^{\circ}(u ; w):=\limsup _{\substack{v \rightarrow u \\ t \downarrow 0}} \frac{\phi(v+t w)-\phi(v)}{t},
$$

from which we can define the Clarke generalized gradient (Clarke subdifferential) [8]:

$$
\partial \phi(u):=\left\{\xi \in \mathbb{R}: \phi^{\circ}(u ; w) \geq \xi w, \forall w \in \mathbb{R}\right\} .
$$

These definitions are given under Assumption 2 of local Lipschitzity of $\phi$. The following proposition characterizes the critical points of $\tilde{g}$.

Proposition 4 (critical points of $\tilde{\mathrm{g}}$ ). Let $a>0, \lambda>0$ and $d \in \mathbb{R}$. Then $\hat{u} \in \mathbb{R}$ is a critical point of $\tilde{\mathrm{g}}$ (i.e. $0 \in \partial \tilde{\mathrm{g}}(\hat{u}))$ if and only if

$$
\begin{cases}a d-a^{2} \hat{u} \in\left[\underline{\delta}^{\hat{u}}, \bar{\delta}^{\hat{u}}\right] & \text { if } \hat{u} \in B \\ a^{2} \hat{u}-a d+\phi^{\prime}(\hat{u})=0 & \text { if } \hat{u} \in \mathbb{R} \backslash B\end{cases}
$$

where

$$
\forall v \in B, \underline{\delta}^{v}=\min \left\{l_{v}^{-}, l_{v}^{+}\right\} \text {and } \bar{\delta}^{v}=\max \left\{l_{v}^{-}, l_{v}^{+}\right\}
$$

with

$$
l_{v}^{-}=\lim _{\substack{u \rightarrow v \\ u<v}} \phi^{\prime}(u) \text { and } l_{v}^{+}=\lim _{\substack{u \rightarrow v \\ u>v}} \phi^{\prime}(u)
$$


Proof. Let consider the case $u \in \mathbb{R} \backslash B$. From [8, corollary to Proposition 2.2.4], $\partial \phi(u)$ is reduced to the singleton $\left\{\phi^{\prime}(u)\right\}$ on a neighbourhood of $u$ if and only if $\phi$ is continuously differentiable on a neighbourhood of $u$. Hence $\partial \phi(u)=\left\{\phi^{\prime}(u)\right\}$ for all $u \in \mathbb{R} \backslash B$ thanks to Assumption 1. Let now consider the case $u \in B$. Then, we have from [8, corollary to Theorem 2.5.1] that

$$
\phi^{\circ}(u ; w)=\limsup _{v \rightarrow u}\left\{\phi^{\prime}(v) w: v \notin B\right\}
$$

Then, this equality together with the definition given in (15), leads to

$$
\begin{aligned}
\xi \in \partial \phi(u) & \Longleftrightarrow \forall w \in \mathbb{R}, \limsup _{v \rightarrow u}\left\{\phi^{\prime}(v) w: v \notin B\right\} \geq \xi w \\
& \Longleftrightarrow\left\{\begin{array}{l}
\forall w \in \mathbb{R}_{+}, w \times \limsup _{v \rightarrow u}\left\{\phi^{\prime}(v): v \notin B\right\} \geq \xi w \\
\forall w \in \mathbb{R}_{-}, w \times \liminf _{v \rightarrow u}\left\{\phi^{\prime}(v): v \notin B\right\} \geq \xi w
\end{array}\right. \\
& \Longleftrightarrow \liminf _{v \rightarrow u}\left\{\phi^{\prime}(v): v \notin B\right\} \leq \xi \leq \limsup _{v \rightarrow u}\left\{\phi^{\prime}(v): v \notin B\right\} \\
& \Longleftrightarrow \min \left\{l_{v}^{-}, l_{v}^{+}\right\} \leq \xi \leq \max \left\{l_{v}^{-}, l_{v}^{+}\right\},
\end{aligned}
$$

for $l_{v}^{-}$and $l_{v}^{+}$given by (18) (note that these limits exist by Assumption 1). Thus, according to the foregoing, we have,

$$
\partial \phi(u)= \begin{cases}{\left[\underline{\delta}^{u}, \bar{\delta}^{u}\right]} & \text { if } u \in B, \\ \left\{\phi^{\prime}(u)\right\} & \text { if } u \in \mathbb{R} \backslash B .\end{cases}
$$

Finally, the differentiability of the quadratic term in (8) leads to

$$
\partial \tilde{\mathrm{g}}(u)=a(a u-d)+\partial \phi(u),
$$

which completes the proof.

From (12) and (16), we are now able to derive conditions on $\phi$ in order to ensure that the global $\operatorname{minimizer}(\mathrm{s}) u^{\star} \in\left\{0, \frac{d}{a}\right\}$ of $\mathrm{g}_{0}$ are critical point(s) of $\tilde{\mathrm{g}}$.

Lemma 5 (global minimizers of $\mathrm{g}_{0}$ are critical points of $\tilde{\mathrm{g}}$ ). Let $a>0$ and $\lambda>0$, then the global minimizer(s) of $\mathrm{g}_{0}$ are critical point(s) of $\tilde{\mathrm{g}}$ for all $d \in \mathbb{R}$ if and only if $\phi$ verifies the two following conditions:

$$
\begin{aligned}
& 0 \in B \text { and } \underline{\delta}^{0} \leq-\sqrt{2 \lambda} a \text { and } \bar{\delta}^{0} \geq \sqrt{2 \lambda} a \\
& B \subset[-\sqrt{2 \lambda} / a, \sqrt{2 \lambda} / a] \text { and } \forall u \in \mathbb{R} \backslash[-\sqrt{2 \lambda} / a, \sqrt{2 \lambda} / a], \phi^{\prime}(u)=0
\end{aligned}
$$

Proof. Let $u_{d}^{\star}$ be a global minimizer of $g_{0}$ for $d \in \mathbb{R} \backslash\{ \pm \sqrt{2 \lambda}\}$. Then, Proposition 3 states that

$$
\forall d \in \mathbb{R},\left\{\begin{array}{l}
|d|<\sqrt{2 \lambda} \Longrightarrow u_{d}^{\star}=0, \\
|d|>\sqrt{2 \lambda} \Longrightarrow u_{d}^{\star}=\frac{d}{a} .
\end{array}\right.
$$

Moreover, from Proposition 4, we have the two following equivalences,

$$
\begin{gathered}
0 \text { is a critical point of } \tilde{\mathrm{g}} \Longleftrightarrow \begin{cases}a d \in\left[\underline{\delta}^{0}, \bar{\delta}^{0}\right] & \text { if } 0 \in B, \\
\phi^{\prime}(0)=a d & \text { if } 0 \in \mathbb{R} \backslash B,\end{cases} \\
\frac{d}{a} \text { is a critical point of } \tilde{\mathrm{g}} \Longleftrightarrow \begin{cases}0 \in\left[\underline{\delta}^{d / a}, \bar{\delta}^{d / a}\right] & \text { if } \frac{d}{a} \in B, \\
\phi^{\prime}\left(\frac{d}{a}\right)=0 & \text { if } \frac{d}{a} \in \mathbb{R} \backslash B .\end{cases}
\end{gathered}
$$


Then, if follows from the previous equations that

$$
\left\{\forall d \in \mathbb{R} \backslash\{ \pm \sqrt{2 \lambda}\}, u^{\star} \text { global minimizer of } \mathrm{g}_{0} \Longrightarrow u^{\star} \text { critical point of } \tilde{\mathrm{g}}\right\}
$$

is equivalent to: $\forall d \in \mathbb{R} \backslash\{ \pm \sqrt{2 \lambda}\}$,

$$
\begin{aligned}
& |d|<\sqrt{2 \lambda} \Longrightarrow \begin{cases}a d \in\left[\underline{\delta}^{0}, \bar{\delta}^{0}\right] & \text { if } 0 \in B, \\
\phi^{\prime}(0)=a d & \text { if } 0 \in \mathbb{R} \backslash B .\end{cases} \\
& |d|>\sqrt{2 \lambda} \Longrightarrow \begin{cases}0 \in\left[\underline{\delta}^{d / a}, \bar{\delta}^{d / a}\right] & \text { if } \frac{d}{a} \in B, \\
\phi^{\prime}\left(\frac{d}{a}\right)=0 & \text { if } \frac{d}{a} \in \mathbb{R} \backslash B .\end{cases}
\end{aligned}
$$

Keeping in mind that (27) holds $\forall d \in \mathbb{R} \backslash\{ \pm \sqrt{2 \lambda}\}$, one can rewrite it as follows:

$$
\begin{cases}(-\sqrt{2 \lambda} a, \sqrt{2 \lambda} a) \subset\left[\underline{\delta}^{0}, \bar{\delta}^{0}\right] & \text { if } 0 \in B, \\ \phi^{\prime}(0)=a d \quad \forall|d|<\sqrt{2 \lambda} & \text { if } 0 \in \mathbb{R} \backslash B .\end{cases}
$$

Clearly, the second line of (29) is impossible for a fixed $\phi$. Hence (27) is equivalent to (22a).

Similarly, one can rewrite (28) as

$$
\forall|u|>\sqrt{2 \lambda} / a, \begin{cases}0 \in\left[\underline{\delta}^{u}, \bar{\delta}^{u}\right] & \text { if } u \in B, \\ \phi^{\prime}(u)=0 & \text { if } u \in \mathbb{R} \backslash B .\end{cases}
$$

The fact that $B$ contains a finite number of points of $\mathbb{R}$ together with the continuity of $\phi$ shows that only the second line of (30) can occur for $|u|>\sqrt{2 \lambda} / a$. Indeed, suppose that there exists $u \in B$ such that $u>\sqrt{2 \lambda} / a$ (one can do the same for $u<-\sqrt{2 \lambda} / a$ ). Then since $B$ is a finite set in $\mathbb{R}$, it contains only isolated points, i.e.

$$
\exists \varepsilon>0, \text { s.t. } B \cap(u-\varepsilon, u+\varepsilon)=\{u\} .
$$

It follows from the second line of (30) that

$$
\forall v \in(\max (\sqrt{2 \lambda} / a, u-\varepsilon), u+\varepsilon) \backslash\{u\}, \phi^{\prime}(v)=0
$$

and that $\phi$ is constant on both $(\max (\sqrt{2 \lambda} / a, u-\varepsilon), u)$ and $(u, u+\varepsilon)$. Then, by continuity, $\phi$ is constant on the whole interval $(\max (\sqrt{2 \lambda} / a, u-\varepsilon), u+\varepsilon)$ contradicting the fact that $u \in B$ (i.e. that $\phi$ is not differentiable at $u$ ). Hence, $B \subset[-\sqrt{2 \lambda} / a, \sqrt{2 \lambda} / a]$ and $\forall u \in \mathbb{R} \backslash[-\sqrt{2 \lambda} / a, \sqrt{2 \lambda} / a], \phi^{\prime}(u)=0$ showing that $(28)$ is equivalent to $(22 \mathrm{~b})$.

Up to now, we have shown that conditions (22) are necessary and sufficient to ensure that global minimizer(s) of $\mathrm{g}_{0}$ are critical point(s) of $\tilde{\mathrm{g}}$ for all $d \in \mathbb{R} \backslash\{ \pm \sqrt{2 \lambda}\}$. To complete the proof, it remains to show that these conditions are sufficient for the case $|d|=\sqrt{2 \lambda}$. Let $d=\sqrt{2 \lambda}$ (the case $d=-\sqrt{2 \lambda}$ is completely symmetric) and (22) be verified. From Proposition 3 both 0 and $\frac{d}{a}$ are global minimizer of $g_{0}$ and from (21) we have

$$
\begin{aligned}
& \partial \tilde{\mathrm{g}}(0)=-a d+\left[\underline{\delta}^{0}, \bar{\delta}^{0}\right]=-\sqrt{2 \lambda} a+\left[\underline{\delta}^{0}, \bar{\delta}^{0}\right] \underset{(22 \mathrm{a})}{\ni} 0 \\
& \begin{cases}\partial \tilde{\mathrm{g}}\left(\frac{d}{a}\right)=\left\{\phi^{\prime}\left(\frac{d}{a}\right)\right\}=\left\{\phi^{\prime}\left(\frac{\sqrt{2 \lambda}}{a}\right)\right\} \underset{(22 \mathrm{~b})}{=}\{0\} & \text { if } \frac{d}{a} \notin B, \\
\partial \tilde{\mathrm{g}}\left(\frac{d}{a}\right)=\left[\underline{\delta}^{d / a}, \bar{\delta}^{d / a}\right]=\left[\underline{\delta}^{\sqrt{2 \lambda} / a}, \bar{\delta}^{\sqrt{2 \lambda} / a}\right] \underset{(17)(22 \mathrm{~b})}{\ni} 0 & \text { if } \frac{d}{a} \in B,\end{cases}
\end{aligned}
$$

since either $\underline{\delta}^{\sqrt{2 \lambda} / a}$ or $\bar{\delta}^{\sqrt{2 \lambda} / a}$ is equal to zero thanks to the facts that $\phi^{\prime}$ is continuous on $\mathbb{R} \backslash B$ and $B$ contains isolated points (Assumption 1). The above equations show that both 0 and $\frac{d}{a}$ are critical points of $\tilde{g}$ which completes the proof. 


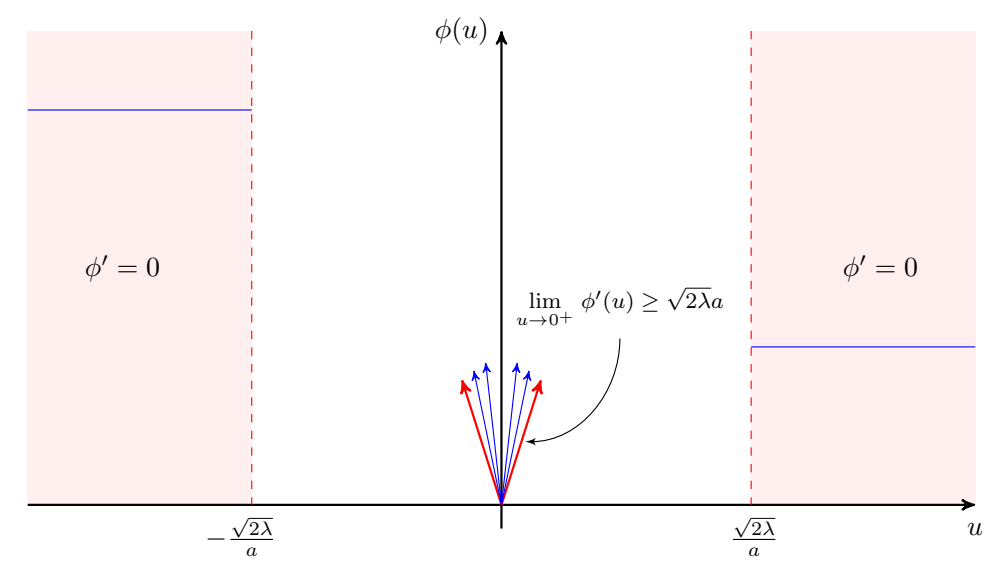

Figure 1: Illustration of the conditions on $\phi$ given by Lemma 5 . The arrows represent condition (22a) (in red) with possible half tangent of $\phi$ at 0 (in blue) and red areas illustrate (22b) where $\phi$ is constant.

Figure 1 illustrates conditions (22).

Lemma 6. Let $a>0$ and $\lambda>0$, then $\tilde{\mathrm{g}}$ do not have any global minimizer $\left(\right.$ s) within $\left(-\frac{\sqrt{2 \lambda}}{a}, 0\right) \cup\left(0, \frac{\sqrt{2 \lambda}}{a}\right)$ for all $d \in \mathbb{R}$ if and only if $\phi$ verifies the following condition:

$$
\forall u \in(-\sqrt{2 \lambda} / a, 0) \cup(0, \sqrt{2 \lambda} / a), \phi(u)>\phi_{\text {сELo }}(a, \lambda ; u),
$$

where $\phi_{\text {CELO }}$ is given in $(6)$.

Proof. Since we have assumed (9), " $\tilde{\mathrm{g}}$ do not have any global minimizer(s) within $\left(-\frac{\sqrt{2 \lambda}}{a}, 0\right) \cup\left(0, \frac{\sqrt{2 \lambda}}{a}\right)$ for all $d \in \mathbb{R} "$ is equivalent to,

$$
\forall u \in(-\sqrt{2 \lambda} / a, 0) \cup(0, \sqrt{2 \lambda} / a), \tilde{\mathrm{g}}(u)>\left\{\begin{array}{ll}
\mathrm{g}_{0}(0)=\frac{d^{2}}{2} & \text { if }|d| \leq \sqrt{2 \lambda}, \\
\mathrm{g}_{0}\left(\frac{d}{a}\right)=\lambda & \text { if }|d| \geq \sqrt{2 \lambda},
\end{array} \forall d \in \mathbb{R}\right.
$$

which can be rewritten as,

$$
\forall u \in(-\sqrt{2 \lambda} / a, 0) \cup(0, \sqrt{2 \lambda} / a), \phi(u)>\max _{d \in \mathbb{R}} f_{u}(d),
$$

where

$$
f_{u}(d)= \begin{cases}-\frac{a^{2} u^{2}}{2}+a u d & \text { if }|d| \leq \sqrt{2 \lambda} \\ \lambda-\frac{1}{2}(a u-d)^{2} & \text { if }|d| \geq \sqrt{2 \lambda}\end{cases}
$$

Then, simple calculations lead to

$$
\max _{|d| \leq \sqrt{2 \lambda}} f_{u}(d)=-\frac{a^{2} u^{2}}{2}+\sqrt{2 \lambda} a|u|
$$

and

$$
\max _{|d| \geq \sqrt{2 \lambda}} f_{u}(d)=\left\{\begin{array}{cl}
\lambda & \text { if }|u| \geq \frac{\sqrt{2 \lambda}}{a} \\
-\frac{a^{2} u^{2}}{2}+\sqrt{2 \lambda} a|u| & \text { otherwise }
\end{array}\right.
$$


Finally we obtain

$$
\forall u \in(-\sqrt{2 \lambda} / a, 0) \cup(0, \sqrt{2 \lambda} / a), \phi(u)>-\frac{a^{2} u^{2}}{2}+\sqrt{2 \lambda} a|u|=\phi_{\text {CELo }}(a, \lambda ; u),
$$

where $\phi_{\text {cELo }}$ is given in (6). This completes the proof.

The following theorem gives necessary and sufficient conditions on $\phi$ in order to have (P1) for $\tilde{g}$.

Theorem 7 (necessary and sufficient conditions for (P1)). Let $a>0$ and $\lambda>0$, then $\tilde{\mathrm{g}}$ has property (P1) (and (9)) for all $d \in \mathbb{R}$ if and only if $\phi$ verifies the three following conditions:

$$
\begin{aligned}
& \phi(0)=0, \\
& \forall u \in \mathbb{R} \backslash(-\sqrt{2 \lambda} / a, \sqrt{2 \lambda} / a), \phi(u)=\lambda|u|_{0}=\lambda, \\
& \forall u \in(-\sqrt{2 \lambda} / a, \sqrt{2 \lambda} / a) \backslash\{0\}, \phi(u)>\phi_{\text {CELO }}(a, \lambda ; u),
\end{aligned}
$$

where $\phi_{\text {CEL }}$ is given in (6).

Proof. We process by showing both implications

$(40) \Rightarrow(\mathrm{P} 1)$ (with (9)): First of all, one can see that $\tilde{g}$ has at least one global minimizer. Indeed, from the continuity of $\phi$ and conditions (40a) and (40b), $\phi$ is bounded. Moreover, the quadratic data fidelity term of $\tilde{\mathrm{g}}$ is coercive $(a>0)$. Thus $\tilde{\mathrm{g}}$ is coercive and its continuity ensures the existence of a global minimizer.

Now, from (40a) and (40b) we get that

$$
\forall u \notin(-\sqrt{2 \lambda} / a, 0) \cup(0, \sqrt{2 \lambda} / a), \phi(u)=\lambda|u|_{0} .
$$

Moreover (40c) ensures with Lemma 6 that $\tilde{\mathrm{g}}$ does not have any global minimizer(s) within $(-\sqrt{2 \lambda} / a, 0) \cup(0, \sqrt{2 \lambda} / a)$ and Proposition 3 shows the same for $g_{0}$. Hence we have shown that $\tilde{\mathrm{g}}$ and $\mathrm{g}_{0}$ are equal at every point which is potentially a global minimizer of one of these two functionals from which we deduce that (P1) holds (with (9)).

$(40) \Leftarrow(\mathrm{P} 1)$ (with $(9))$ : Under $(\mathrm{P} 1)$, all the global minimizer(s) of $\mathrm{g}_{0}$ are critical points of $\tilde{\mathrm{g}}$ which, from Lemma 5 , is equivalent to (22). Moreover (9) leads to (40a) and allows to reduce (22b) to (40b). Finally, assume that (40c) does not hold and that there exists $u_{0} \in(0, \sqrt{2 \lambda} / a)$ such that $\phi\left(u_{0}\right) \leq \phi_{\text {сво }}\left(u_{0}\right)$. Then, one can easily get a $d_{0}=\sqrt{2 \lambda}$ for which g gas two global minimizers $\left\{0, \frac{d_{0}}{a}\right\}$ and for which the whole interval $\left[0, \frac{d_{0}}{a}\right]=\left[0, \frac{\sqrt{2 \lambda}}{a}\right]$ minimizes the CEL0 functional $\mathrm{g}_{\text {cELo }}$ (defined by (8) for $\phi=\phi_{\text {сЕц }}$ ) since $\mathrm{g}_{\text {свL }}$ is the convex hull of $\mathrm{g}_{0}[26$, Section 2]. Hence, we have $u_{0} \notin\left\{0, \frac{d_{0}}{a}\right\}$ and nevertheless $\tilde{\mathrm{g}}\left(u_{0}\right) \leq \mathrm{g}_{\text {CEL }}\left(u_{0}\right)=\mathrm{g}_{0}(0)=\mathrm{g}_{0}\left(\frac{d_{0}}{a}\right)$ which contradicts (P1) and completes the proof.

Remark 8. One can notice that (40c) imposes $\phi$ to be singular at the origin. We highlight here a well-known property stating that sparsity is enforced by penalties singular at the origin. Moreover condition (40b) leads to penalties which are constant for large $|u|$. This was known to be a condition for unbiasedness [11].

A schema showing conditions (40) is presented on Figure 2. One can see that within the gray zone, the penalty can be totally arbitrary as long as it is continuous, equal to zero at the origin and equal to $\lambda$ at $\pm \sqrt{2 \lambda} / a$. Moreover, for $|u| \geq \sqrt{2 \lambda} / a$, the penalty must be constant equal to $\lambda$.

Now, we shall ensure that each (local) minimizer of $\tilde{g}$ is a (local) minimizer of $g_{0}$. The following theorem states necessary and sufficient conditions on $\phi$ to have both (P1) and (P2) for $\tilde{g}$. 


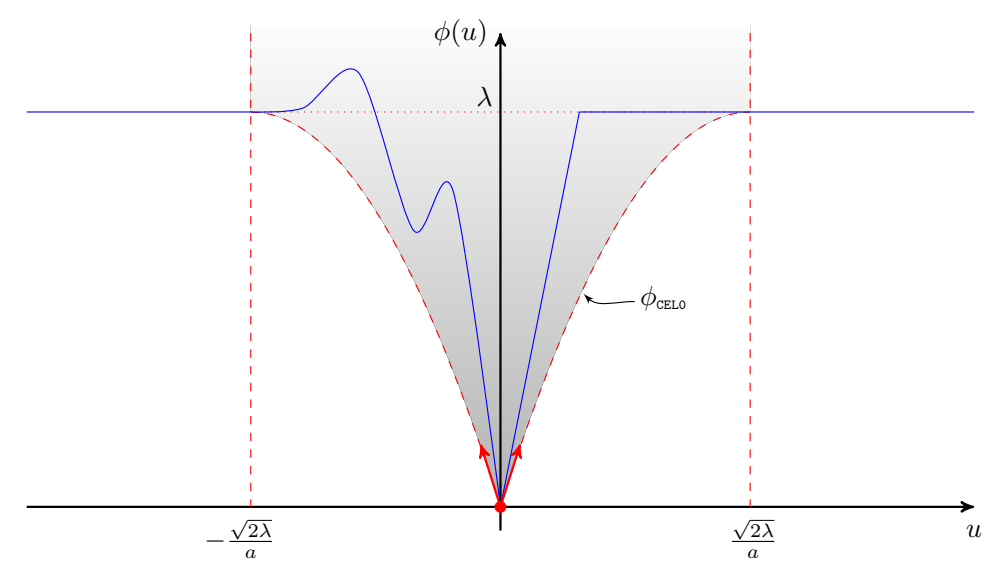

Figure 2: Illustration of the conditions given by Theorem 7. Dashed red curves together with the red point at 0 represent conditions (40). The gray zone is the admissible part of the plan where the penalty must verify condition (40c). An example of such a penalty is showed in blue.

Theorem 9 (necessary and sufficient conditions for (P1) and (P2)). Let $a>0$ and $\lambda>0$, then $\tilde{\mathrm{g}}$ has both properties $(\mathrm{P} 1)$ and $(\mathrm{P} 2)$ for all $d \in \mathbb{R}$ if and only if, in addition to conditions (40), $\phi$ verifies the two following conditions:

$$
\begin{aligned}
& \forall u \in B \backslash\{0\}, \lim _{\substack{v \rightarrow u \\
v<u}} \phi^{\prime}(v)>\lim _{\substack{v \rightarrow u \\
v>u}} \phi^{\prime}(v) \\
& \forall u \in\left(\beta^{-}, \beta^{+}\right) \backslash B,\left\{\begin{array}{l}
\phi^{\prime \prime}(u) \leq-a^{2} \text { and } \\
\forall \varepsilon>0, \exists v_{\varepsilon} \in(u-\varepsilon, u+\varepsilon) \text { s.t. } \phi^{\prime \prime}\left(v_{\varepsilon}\right)<-a^{2}
\end{array}\right.
\end{aligned}
$$

for $\beta^{-} \in[-\sqrt{2 \lambda} / a, 0)$ and $\beta^{+} \in(0, \sqrt{2 \lambda} / a]$ defined as the larger (resp. lower) real for which $\phi$ is constant on the whole interval $\left(-\infty, \beta^{-}\right]$(resp. $\left.\left[\beta^{+},+\infty\right)\right)$. With this definition, $B \subset\left[\beta^{-}, \beta^{+}\right]$.

Proof. The proof is given in Appendix A.

Remark 10. For the CELO penalty (6), condition (42b) is not verified. Indeed we have,

$$
\forall u \in\left(-\frac{\sqrt{2 \lambda}}{a}, \frac{\sqrt{2 \lambda}}{a}\right) \backslash\{0\}, \phi_{\text {CELO }}^{\prime \prime}(a, \lambda ; u)=-a^{2},
$$

However, in this case, when $\phi_{\mathrm{CEL} 0}^{\prime}(a, \lambda ; u)=a d-a^{2} u \Leftrightarrow|a d|=\sqrt{2 \lambda} a$ (by definition of CELO), the whole interval $\left[0, \frac{\sqrt{2 \lambda}}{a}\right]$ (resp. $\left[-\frac{\sqrt{2 \lambda}}{a}, 0\right]$ depending on the sign of the quantity ad) minimizes $\tilde{\mathrm{g}}$ and one can easily get a minimizer of $\mathrm{g}_{0}$ by simple thresholding [26]. Moreover, condition (40c) is also not verified by the CELO penalty. Finally, from conditions (40) and (42), the CELO penalty can be seen as the inferior limit of the resulting class of penalties.

Remark 11. Under the conditions of Theorems 7 and 9, $\phi$ is strictly concave-decreasing on $\left[\beta^{-}, 0\right]$ (resp. strictly concave-increasing on $\left.\left[0, \beta^{+}\right]\right)$. Hence, from $(40 \mathrm{c})$, if $\beta^{-}=-\frac{\sqrt{2 \lambda}}{a}$ (resp. $\beta^{+}=\frac{\sqrt{2 \lambda}}{a}$ ) then necessarily $\beta^{-} \notin B$ (resp. $\beta^{+} \notin B$ ) and $\phi^{\prime}\left(\beta^{-}\right)=0$ (resp. $\left.\phi^{\prime}\left(\beta^{+}\right)=0\right)$. See Figure 3 for an illustration.

Finally, Theorems 7 and 9 provide necessary and sufficient conditions on $\phi$ in order to make the continuous relaxation $\tilde{g}$ verifying properties $(\mathrm{P} 1)$ and $(\mathrm{P} 2)$. These conditions are illustrated on Figure 3. In the following of the paper, we extend these results to the $N$-dimensional case. 


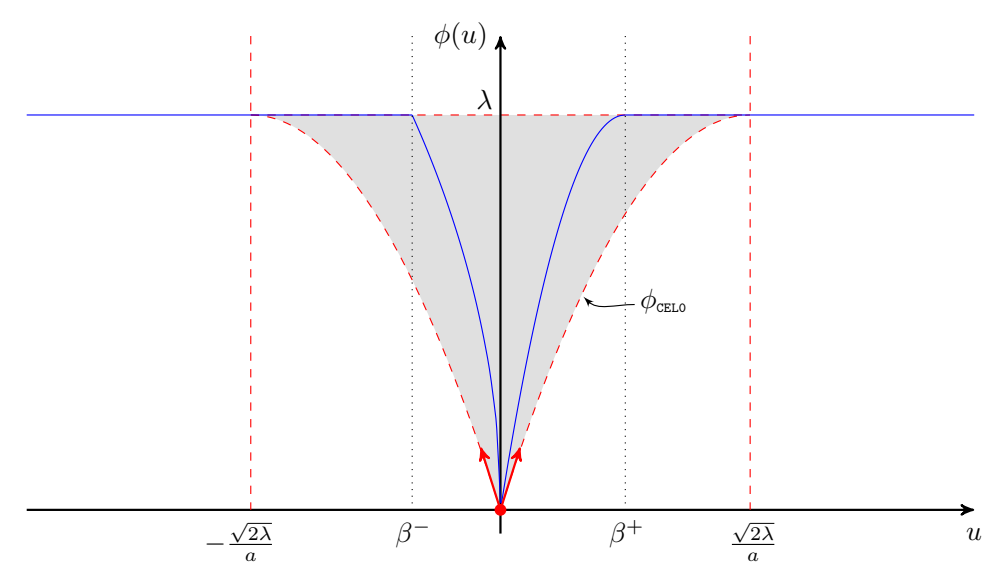

Figure 3: Illustrations of all the conditions given by Theorems 7 and 9 to have (P1) and (P2). Dashed red curves together with the red point at 0 represent the conditions while the blue curve shows an example of exact penalty verifying such conditions. Between $\beta^{-}$and $\beta^{+}$, the penalty must stay in the gray area and verify conditions (42).

\section{When $A$ has nonzero orthogonal columns}

Let's consider $\mathrm{G}_{\ell_{0}}$ defined in (1) and $\tilde{\mathrm{G}}$ in (4). Then, following [26, Section 3], the case where the matrix $A$ has nonzero orthogonal columns (i.e. $A^{T} A$ is diagonal) can easily be deduced from the previous 1D study. Note that in this case we necessarily have $M \geq N$. Let $\hat{d}=A D^{-2} A^{T} d$ and $\tilde{z}=D^{-1} A^{T} d$, where $D \in \mathbb{R}^{N \times N}$ is a diagonal matrix with diagonal elements $d_{i}=\left\|a_{i}\right\| \forall i \in \mathbb{I}_{N}\left(D^{-1}\right.$ is well defined since we consider matrices such that $\left\|a_{i}\right\|>0$ for all $i$ ), then we have

$$
\frac{1}{2}\|A \mathrm{x}-d\|^{2}=\frac{1}{2}\|d-\hat{d}\|^{2}+\frac{1}{2}\|D \mathrm{x}-\tilde{z}\|^{2} .
$$

Using this relation, $\mathrm{G}_{\ell_{0}}$ can be rewritten as,

$$
\mathrm{G}_{\ell_{0}}(\mathrm{x})=\frac{1}{2}\|d-\hat{d}\|^{2}+\sum_{i \in \mathbb{I}_{N}} \frac{1}{2}\left(\left\|a_{i}\right\| \mathrm{x}_{i}-\tilde{z}_{i}\right)^{2}+\lambda\left|\mathrm{x}_{i}\right|_{0}
$$

and its minimization is reduced to the minimization of $N$ independent $1 \mathrm{D}$ functionals. According to Theorems 7 and 9, necessary and sufficient conditions on $\Phi$ in order to have (P1) and (P2) for all $d \in \mathbb{R}^{M}$, are given by: $\forall i \in \mathbb{I}_{N}$,

$$
\begin{aligned}
& \phi_{i}(0)=0, \\
& \exists \beta^{i-} \in\left[-\frac{\sqrt{2 \lambda}}{\left\|a_{i}\right\|}, 0\right) \text { and } \beta^{i+} \in\left(0, \frac{\sqrt{2 \lambda}}{\left\|a_{i}\right\|}\right] \text { s.t. } \forall u \in \mathbb{R} \backslash\left(\beta^{i-}, \beta^{i+}\right), \phi_{i}(u)=\lambda, \\
& \forall u \in\left(\beta^{i-}, \beta^{i+}\right) \backslash\{0\}, \phi_{i}(u)>\phi_{\text {CELo }}\left(\left\|a_{i}\right\|, \lambda ; u\right), \\
& \forall u \in B^{i} \backslash\{0\}, \lim _{\substack{v \rightarrow u \\
v<u}} \phi_{i}^{\prime}(v)>\lim _{\substack{v \rightarrow u \\
v>u}} \phi_{i}^{\prime}(v), \\
& \forall u \in\left(\beta^{i-}, \beta^{i+}\right) \backslash B^{i},\left\{\begin{array}{l}
\phi_{i}^{\prime \prime}(u) \leq-\left\|a_{i}\right\|^{2} \text { and } \\
\forall \varepsilon>0, \exists v_{\varepsilon} \in(u-\varepsilon, u+\varepsilon) \text { s.t. } \phi_{i}^{\prime \prime}\left(v_{\varepsilon}\right)<-\left\|a_{i}\right\|^{2}
\end{array}\right.
\end{aligned}
$$


where $B^{i} \ni 0$ is a finite subset of $\left[\beta^{i-}, \beta^{i+}\right]$ on which $\phi_{i}$ is not differentiable. Let us recall that the $\mathrm{N}$-dimensional penalty $\Phi$ is given by,

$$
\Phi(\mathrm{x})=\sum_{i \in \mathbb{I}_{N}} \phi_{i}\left(\mathrm{x}_{i}\right) .
$$

The following proposition gives a relation between conditions (46) which can be useful in practice to define penalties verifying the five conditions (46).

Proposition 12. Let $i \in \mathbb{I}_{N}$ and $B^{i} \subseteq\left\{\beta^{i-}, 0, \beta^{i+}\right\}$, then the following implication holds:

$$
\{(46 \mathrm{a}),(46 \mathrm{~b}),(46 \mathrm{e})\} \Longrightarrow(46 \mathrm{c}) \text {. }
$$

Proof. Let $\phi_{i}$ verifying conditions (46a), (46b), (46e) and $f=\phi_{i}-\phi_{\text {cЕLo }}\left(\left\|a_{i}\right\|, \lambda ; \cdot\right)$. Then we have $f(0)=0$ and $f\left(\beta^{i+}\right)=\lambda-\phi_{\text {свьо }}\left(\left\|a_{i}\right\|, \lambda ; \beta^{i+}\right) \geq 0$. Moreover, by assumption on $B^{i} \subseteq\left\{\beta^{i-}, 0, \beta^{i+}\right\}, f$ is twice differentiable on $\left(0, \beta^{i+}\right)$ and $\forall u \in\left(0, \beta^{i+}\right)$,

$$
\left\{\begin{array}{l}
f^{\prime \prime}(u)=\phi_{i}^{\prime \prime}(u)+\left\|a_{i}\right\|^{2} \leq 0 \text { and } \\
\forall \varepsilon>0, \exists v_{\varepsilon} \in(u-\varepsilon, u+\varepsilon) \text { s.t. } f^{\prime \prime}\left(v_{\varepsilon}\right)=\phi_{i}^{\prime \prime}\left(v_{\varepsilon}\right)+\left\|a_{i}\right\|^{2}<0
\end{array},\right.
$$

showing that $f^{\prime \prime}(u)=0$ only for isolated points in $\left(0, \beta^{i+}\right)$. Hence $f$ is strictly concave on $\left(0, \beta^{i+}\right)$ implying that $\forall u \in\left(0, \beta^{i+}\right) \phi_{i}(u)>\phi_{\text {CELo }}\left(\left\|a_{i}\right\|, \lambda ; u\right)$. Same arguments can be used to show the result on $\left(\beta^{i-}, 0\right)$.

According to Section 2, while the five conditions (46) are necessary and sufficient to have $\{(\mathrm{P} 1),(\mathrm{P} 2)\}$ for $\tilde{\mathrm{G}}$ and $\mathrm{G}_{\ell_{0}}$ (Theorem 9), only conditions (46a)-(46c) are required to only have (P1) (Theorem 7). The main question is then to know if conditions (46) remain valid for an arbitrary $A \in \mathbb{R}^{M \times N}$.

\section{Extension to an arbitrary matrix $A \in \mathbb{R}^{M \times N}$}

This section presents the main result of the paper. We show that conditions (46) are necessary and sufficient to have (P1) and (P2) for $\mathrm{G}_{\ell_{0}}$, defined in (1), and $\tilde{\mathrm{G}}$, defined in (4), without any assumption on the matrix $A \in \mathbb{R}^{M \times N}$ (except $\left\|a_{i}\right\|>0$ for all $i$ ). First of all, since the case where $A$ has nonzero orthogonal columns is a special case of $A \in \mathbb{R}^{M \times N}$, we get from the previous section that if we do not consider any assumption on $A \in \mathbb{R}^{M \times N}$,

- conditions (46) are necessary to have $\{(\mathrm{P} 1),(\mathrm{P} 2)\}$,

- only conditions (46a), (46b) and (46c) are necessary to have (P1).

Note that one may define weaker conditions for specific $A \in \mathbb{R}^{M \times N}$ and $d \in \mathbb{R}^{M}$. However, in the present paper, we are concerned with conditions valid for any $d \in \mathbb{R}^{M}$ and which do not require a special structure of the matrix $A \in \mathbb{R}^{M \times N}$ (but these conditions may be expressed in function of the elements of $A$ ). Then, the goal in what follows is to show that conditions (46) are also sufficient.

Theorem 13 (links between global minimizers of $\tilde{\mathrm{G}}$ and $\mathrm{G}_{\ell_{0}}$ ). Let $A \in \mathbb{R}^{M \times N}, \lambda>0$ and $\tilde{\mathrm{G}}$ be defined with $\Phi$ verifying conditions (46a), (46b) and (46c). Then, $\forall d \in \mathbb{R}^{M}$, the two following statements hold true:

$$
\begin{aligned}
& \text { 1. } \arg \min _{\mathrm{x} \in \mathbb{R}^{N}} \mathrm{G}_{\ell_{0}}(\mathrm{x})=\arg \min _{\mathrm{x} \in \mathbb{R}^{N}} \tilde{\mathrm{G}}(\mathrm{x}), \\
& \text { 2. } \min _{\mathrm{x} \in \mathbb{R}^{N}} \mathrm{G}_{\ell_{0}}(\mathrm{x})=\min _{\mathrm{x} \in \mathbb{R}^{N}} \tilde{\mathrm{G}}(\mathrm{x}) .
\end{aligned}
$$


Proof. From, (46a), (46b) and (46c), it is clear that,

$$
\forall \mathrm{x} \in \mathbb{R}^{N}, \mathrm{G}_{\mathrm{CELL}}(\mathrm{x}) \leq \tilde{\mathrm{G}}(\mathrm{x}) .
$$

Let $\hat{\mathrm{x}}$ be a global minimizer of $\mathrm{G}_{\ell_{0}}$, then from [20, Proposition 4.1] we have

$$
\forall i \in \sigma(\hat{\mathrm{x}}), \hat{\mathrm{x}}_{i} \in \mathbb{R} \backslash\left(-\frac{\sqrt{2 \lambda}}{\left\|a_{i}\right\|}, \frac{\sqrt{2 \lambda}}{\left\|a_{i}\right\|}\right)
$$

where $\sigma(\hat{\mathrm{x}})$ stands for the support of $\hat{\mathrm{x}}$. This implies, from (46a) and (46b),

$$
\mathrm{G}_{\ell_{0}}(\hat{\mathrm{x}})=\mathrm{G}_{\mathrm{CELO}}(\hat{\mathrm{x}})=\tilde{\mathrm{G}}(\hat{\mathrm{x}}) \text {. }
$$

Then, [26, Theorem 4.5 (i)] ensures that $\hat{x}$ is also a global minimizer of $\mathrm{G}_{\text {CËо }}$ which, with (51) and (53), proves the inclusion $\arg \min _{\mathrm{x}} \mathrm{G}_{\ell_{0}}(\mathrm{x}) \subseteq \arg \min _{\mathrm{x}} \tilde{\mathrm{G}}(\mathrm{x})$ in (49). Thus, there is at least one point, denoted $\mathrm{x}^{\star} \in \mathbb{R}^{N}$, which is a global minimizer of the three functionals (existence of minimizers for $\mathrm{G}_{\ell_{0}}$ is established in [20, Theorem 4.4 (i)]). Let now $\hat{\mathrm{x}} \in \mathbb{R}^{N}$ be a global minimizer of $\tilde{\mathrm{G}}$. Clearly, $\tilde{\mathrm{G}}(\hat{\mathrm{x}})=$ $\tilde{\mathrm{G}}\left(\mathrm{x}^{\star}\right)=\mathrm{G}_{\text {CELO }}\left(\mathrm{x}^{\star}\right)$ and since $\mathrm{G}_{\text {CELO }} \leq \tilde{\mathrm{G}}$ (eq. $\left.(51)\right)$, $\hat{\mathrm{x}}$ is also a global minimizer of $\mathrm{G}_{\text {CELO }}$ and $\tilde{\mathrm{G}}(\hat{\mathrm{x}})=\mathrm{G}_{\text {CELO }}(\hat{\mathrm{x}})$. Combining this last equality with (46c) leads to

$$
\forall i \in \sigma(\hat{\mathrm{x}}), \hat{\mathrm{x}}_{i} \notin\left(\beta^{i-}, \beta^{i+}\right),
$$

and it comes from (46a) and (46b) that $\mathrm{G}_{\ell_{0}}(\hat{\mathrm{x}})=\tilde{\mathrm{G}}(\hat{\mathrm{x}})=\tilde{\mathrm{G}}\left(\mathrm{x}^{\star}\right)=\mathrm{G}_{\ell_{0}}\left(\mathrm{x}^{\star}\right)$. Thus, $\hat{\mathrm{x}}$ is a global minimizer of $\mathrm{G}_{\ell_{0}}$ which proves the inclusion $\arg \min _{\mathrm{x}} \mathrm{G}_{\ell_{0}}(\mathrm{x}) \supseteq \arg \min _{\mathrm{x}} \tilde{\mathrm{G}}(\mathrm{x})$ in (49). Equality (50) is straightforward from the foregoing.

Hence, Theorem 13 shows that conditions (46a), (46b) and (46c) are sufficient to have (P1). Moreover, we can deduce from this result the existence of minimizers for $\tilde{G}$ as stated by the following proposition.

Proposition 14 (existence of global minimizers for $\tilde{\mathrm{G}}$ ). Let $\tilde{\mathrm{G}}$ be defined as in Theorem 13. Then, the set of global minimizers of $\tilde{\mathrm{G}}$ is nonempty.

Proof. From [20, Theorem 4.4 (i)], the set of global minimizers of $\mathrm{G}_{\ell_{0}}$ is nonempty. Then, the result for $\tilde{\mathrm{G}}$ is straightforward from Theorem 13 .

In order to analyse the links between (local) minimizers of $\tilde{\mathrm{G}}$ and $\mathrm{G}_{\ell_{0}}$, we start by showing two preliminary results.

Proposition 15. Let $A \in \mathbb{R}^{M \times N}, \lambda>0$ and $\tilde{\mathrm{G}}$ be defined with $\Phi$ verifying conditions (46b), (46d) and (46e). Then $\forall i \in \mathbb{I}_{N}, t \mapsto \tilde{\mathrm{G}}\left(\mathrm{x}^{(i)}+e_{i} t\right)$, the restriction of $\tilde{\mathrm{G}}$ to the ith variable at point $\mathrm{x} \in \mathbb{R}^{N}$, is strictly concave on $\left(\beta^{i-}, 0\right)$ and on $\left(0, \beta^{i+}\right)$ and strictly convex beyond.

Proof. Let $i \in \mathbb{I}_{N}$ and consider the restriction of $\tilde{\mathrm{G}}$ to the ith variable at point $\mathrm{x} \in \mathbb{R}^{N}$,

$$
f(t)=\tilde{\mathrm{G}}\left(\mathrm{x}^{(i)}+e_{i} t\right)=\frac{\left\|a_{i}\right\|^{2}}{2} t^{2}+t\left\langle a_{i}, A \mathrm{x}^{(i)}-d\right\rangle+\phi_{i}(t)+C,
$$

where $\mathrm{x}^{(i)}=\left(\mathrm{x}_{1}, \ldots, \mathrm{x}_{i-1}, 0, \mathrm{x}_{i+1}, \ldots, \mathrm{x}_{N}\right)$ and $C=\frac{1}{2}\left\|A \mathrm{x}^{(i)}-d\right\|^{2}+\sum_{j \in \mathbb{I}_{N} \backslash\{i\}} \phi_{j}\left(\mathrm{x}_{j}\right)$ is a constant independent of $t$. Then, from (46e), one can easily get that

$$
\forall t \in\left(\beta^{i-}, \beta^{i+}\right) \backslash B^{i},\left\{\begin{array}{l}
f^{\prime \prime}(t)=\phi_{i}^{\prime \prime}(t)+\left\|a_{i}\right\|^{2} \leq 0 \text { and } \\
\forall \varepsilon>0, \exists v_{\varepsilon} \in(t-\varepsilon, t+\varepsilon) \text { s.t. } f^{\prime \prime}\left(v_{\varepsilon}\right)=\phi_{i}^{\prime \prime}\left(v_{\varepsilon}\right)+\left\|a_{i}\right\|^{2}<0
\end{array}\right.
$$

Moreover (46d) leads to

$$
\forall t \in B^{i} \backslash\{0\}, \lim _{\substack{u \rightarrow t \\ u<t}} f^{\prime}(u)>\lim _{\substack{u \rightarrow t \\ u>t}} f^{\prime}(u) .
$$

These two results prove the strict concavity of $f$ on $\left(\beta^{i-}, 0\right)$ and $\left(0, \beta^{i+}\right)$. Then, it comes from (46b) that $\forall t \in\left(-\infty, \beta^{i-}\right) \cup\left(\beta^{i+},+\infty\right) f^{\prime \prime}(t)=\left\|a_{i}\right\|^{2}>0$ which completes the proof. 
A consequence of Proposition 15 is given by the following result.

Proposition 16. Let $\tilde{\mathrm{G}}$ be defined as in Proposition 15. If $\tilde{\mathrm{G}}$ reaches a (local) minimum at $\hat{\mathrm{x}} \in \mathbb{R}^{N}$, then

$$
\forall i \in \sigma(\hat{\mathrm{x}}), \quad \hat{\mathrm{x}}_{i} \in\left(-\infty, \beta^{i-}\right] \cup\left[\beta^{i+},+\infty\right) .
$$

Moreover, if $\beta^{i-} \in B^{i}$ (resp. $\left.\beta^{i+} \in B^{i}\right)$, then $\left(-\infty, \beta^{i-}\right]$ (resp. $\left[\beta^{i+},+\infty\right)$ ) in (56) can be reduced to $\left(-\infty, \beta^{i-}\right)\left(\operatorname{resp} .\left(\beta^{i+},+\infty\right)\right)$.

Proof. The proof of (56) is straightforward from Proposition 15 which states that the restriction of $\tilde{\mathrm{G}}$ to the ith variable, is strictly concave on $\left(\beta^{i-}, 0\right)$ and on $\left(0, \beta^{i+}\right)$. Then, the fact that we can consider open intervals when $\beta^{i-} \in B^{i}$ (resp. $\beta^{i+} \in B^{i}$ ) comes from the same arguments as the ones used in the proof of Proposition 15 for points belonging to $B^{i}$.

Remark 17. It is shown in [26, Section 4.2.2] that local (not global) minimizers of $\mathrm{G}_{\ell_{0}}$ for which $\exists i \in \sigma(\hat{\mathrm{x}})$ such that $\left|\hat{\mathrm{x}}_{i}\right|<\frac{\sqrt{2 \lambda}}{\left\|a_{i}\right\|}$ are eliminated with the CELO functional. Proposition 16 extends this result to $\tilde{\mathrm{G}}$ (if conditions (46b), (46d) and (46e) hold) and shows that such a $\tilde{\mathrm{G}}$ eliminates minimizers $\hat{\mathrm{x}}$ of $\mathrm{G}_{\ell_{0}}$ for which

$$
\exists i \in \sigma(\hat{\mathrm{x}}) \text { s.t. } \hat{\mathrm{x}}_{i} \in\left(\beta^{i-}, \beta^{i+}\right) .
$$

Hence, from (46b), $\tilde{\mathrm{G}}$ potentially eliminates fewer local minimizers of $\mathrm{G}_{\ell_{0}}$ than $\mathrm{G}_{\mathrm{CEL}}$.

We are now able to derive the following result between (local) minimizers of $\tilde{\mathrm{G}}$ and $\mathrm{G}_{\ell_{0}}$.

Theorem 18 (links between (local) minimizers of $\tilde{\mathrm{G}}$ and $\mathrm{G}_{\ell_{0}}$ ). Let $A \in \mathbb{R}^{M \times N}, \lambda>0$ and $\tilde{\mathrm{G}}$ be defined with $\Phi$ verifying conditions (46). Then, $\forall d \in \mathbb{R}^{M}$, the two following statements hold true:

1. $\hat{\mathrm{x}}$ (local) minimizer of $\tilde{\mathrm{G}} \Longrightarrow \hat{\mathrm{x}}$ (local) minimizer of $\mathrm{G}_{\ell_{0}}$,

2. $\tilde{\mathrm{G}}(\hat{\mathrm{x}})=\mathrm{G}_{\ell_{0}}(\hat{\mathrm{x}})$.

Proof. Let $\hat{\mathrm{x}} \in \mathbb{R}^{N}$ be a (local) minimizer of $\tilde{\mathrm{G}}$ and $\hat{\sigma}=\sigma(\hat{\mathrm{x}})$. Thus we have $0_{\mathbb{R}^{N}} \in \partial \tilde{\mathrm{G}}(\hat{\mathrm{x}})$ which is a necessary condition for a point to be a local optimum of $\tilde{G}$ [8]. Since the quadratic term in $\tilde{\mathrm{G}}$ is differentiable we have,

$$
\forall \mathrm{x} \in \mathbb{R}^{N}, \partial \tilde{\mathrm{G}}(\mathrm{x})=A^{T}(A \mathrm{x}-d)+\partial \Phi(\mathrm{x})=\prod_{i \in \mathbb{I}_{N}}\left[A^{T}(A \mathrm{x}-d)\right]_{i}+\partial \phi_{i}\left(\mathrm{x}_{i}\right) .
$$

From Proposition 16, $\hat{x}$ verifies (56) which, combined with conditions (46b) (46c) leads to

$$
0_{\mathbb{R}^{N}} \in \partial \tilde{\mathrm{G}}(\hat{\mathrm{x}}) \Longleftrightarrow \forall i \in \mathbb{I}_{N}, \begin{cases}\left\langle a_{i}, d-A \hat{\mathrm{x}}\right\rangle \in\left[\underline{\delta}_{i}^{0}, \bar{\delta}_{i}^{0}\right] & \text { if } i \notin \hat{\sigma} \\ \left\langle a_{i}, d-A \hat{\mathrm{x}}\right\rangle=0 & \text { if } i \in \hat{\sigma} .\end{cases}
$$

where $\underline{\delta}_{i}^{0}$ and $\bar{\delta}_{i}^{0}$ are defined as in (17) for $\phi_{i}$. Then, the second line of (61) can be rewritten as follows:

$$
\left(A_{\hat{\sigma}}\right)^{T} A_{\hat{\sigma}} \hat{\mathrm{x}}_{\hat{\sigma}}=\left(A_{\hat{\sigma}}\right)^{T} d,
$$

which ensures that $\hat{\mathrm{x}}$ is a (local) minimizer of $\mathrm{G}_{\ell_{0}}$ [20, Corollary 2.5]. Finally, equality (59) comes from Proposition 16 and conditions (46a)(46b).

Theorem 18 is thus the counterpart of Theorem 13 for property (P2) showing that conditions (46) are sufficient to have (P2) between $\tilde{\mathrm{G}}$ and $\mathrm{G}_{\ell_{0}}$. 
Finally, we have shown in this section that the three first conditions in (46) are necessary and sufficient to have $(\mathrm{P} 1)$ while all conditions $(46)$ are required to have $\{(\mathrm{P} 1),(\mathrm{P} 2)\}$. Hence, conditions $(46)$ define a class of exact continuous penalties approximating the $\ell_{0}$-norm for problem (1). As outlined in Remark 10, the CEL0 penalty is the inferior limit of this class of functions. Then, for all penalties belonging to this class, the resulting functional $\tilde{\mathrm{G}}$ eliminates some local (not global) minimizers of $\mathrm{G}_{\ell_{0}}$. However, as stated by Remark 17, the amount of removed local (not global) minimizers is potentially lower than the one corresponding to $\mathrm{G}_{\mathrm{CEL} \text { O }}$. Moreover, $\mathrm{G}_{\mathrm{CELO}}$ is the only one leading to the convex hull of $\mathrm{G}_{\ell_{0}}$ in the case where the matrix $A$ has nonzero orthogonal columns [26] and being convex with respect to each component for any matrix $A \in \mathbb{R}^{M \times N}$. The limiting penalty $\Phi_{\text {CELo }}$ seems thus to be the most suited to continuously approximate the $\ell_{0}$-norm in problem (1) (see [26] for details about the special case CEL0).

\section{State of the art penalties analysis}

This part is devoted to the analysis of state of the art penalties in the proposed framework of exact continuous relaxations of the $\ell_{0}$-norm. Thanks to conditions (46), we highlight bounds on the parameters defining such penalties ensuring that the resulting relaxation $\tilde{\mathrm{G}}$ verifies $(\mathrm{P} 1)$ or $\{(\mathrm{P} 1),(\mathrm{P} 2)\}$.

\subsection{Capped- $\ell_{1}$}

The Capped- $\ell_{1}$ (or truncated- $\ell_{1}$ ) penalty [33] is defined by

$$
\Phi_{\text {cap }}(\mathrm{x}):=\sum_{i \in \mathbb{I}_{N}} \lambda \min \left\{\theta_{i}\left|\mathrm{x}_{i}\right|, 1\right\}
$$

where $\theta_{i} \in \mathbb{R}_{+}^{\star}$ for all $i \in \mathbb{I}_{N}$.

As stated in the following proposition, by choosing properly the parameters $\theta_{i}\left(i \in \mathbb{I}_{N}\right)$, one can ensures that the relaxed functional $\mathrm{G}_{\text {cap }}$ verifies property $(\mathrm{P} 1)$.

Proposition 19. Property (P1) holds for $\mathrm{G}_{\mathrm{Cap}}$ if and only if

$$
\forall i \in \mathbb{I}_{N}, \lambda \theta_{i} \geq \sqrt{2 \lambda}\left\|a_{i}\right\|
$$

Moreover, $\mathrm{G}_{\mathrm{Cap}}$ cannot verify (P2).

Proof. By definition of $\Phi_{\text {cap }}$, condition (46a) is verified for all $\theta_{i}>0$. Then, one can easily see that for the Capped- $\ell_{1}$ penalty, we have

$$
\forall i \in \mathbb{I}_{N}, \beta^{i-}=-\frac{1}{\theta_{i}} \text { and } \beta^{i+}=\frac{1}{\theta_{i}} .
$$

Then,

$$
(64) \Longrightarrow \forall i \in \mathbb{I}_{N}, \frac{1}{\theta_{i}} \leq \frac{\sqrt{\lambda}}{\sqrt{2}\left\|a_{i}\right\|} \leq \frac{\sqrt{2 \lambda}}{\left\|a_{i}\right\|} \underset{\overline{(65)}}{\Longrightarrow}(46 \mathrm{~b})
$$

Moreover,

$$
\forall i \in \mathbb{I}_{N}, \lim _{u \rightarrow 0^{+}} \phi_{\text {cap }}^{\prime}\left(\theta_{i}, \lambda ; u\right)=\lambda \theta_{i} \text { and } \lim _{u \rightarrow 0^{-}} \phi_{\text {cap }}^{\prime}\left(\theta_{i}, \lambda ; u\right)=-\lambda \theta_{i},
$$

where $\phi_{\text {cap }}(\theta, \lambda ; u)=\lambda \min \{\theta|u|, 1\}$ for $u \in \mathbb{R}, \theta \in \mathbb{R}_{+}^{\star}$ and $\lambda \in \mathbb{R}_{+}^{\star}$.

Then, the fact that $\phi_{\text {CELo }}^{\prime}\left(\left\|a_{i}\right\|, \lambda ; u\right) \rightarrow \sqrt{2 \lambda}\left\|a_{i}\right\|$ as $u \rightarrow 0^{+}$(resp. $-\sqrt{2 \lambda}\left\|a_{i}\right\|$ as $u \rightarrow 0^{-}$) together with $(67)$ and the fact that, on $\left[\beta^{-}, 0\right]$ and $\left[0, \beta^{+}\right], \phi_{\text {Cap }}$ is linear and $\phi_{\text {CELo }}$ strictly concave, shows that $(46 \mathrm{c}) \Longleftrightarrow(64)$. Finally, one can easily see that (46e) cannot be satisfied since $\phi_{\text {cap }}$ is linear on $\left[\beta^{-}, 0\right]$ and $\left[0, \beta^{+}\right]$. 
Figure 4 shows Capped- $\ell_{1}$ penalties for which $\theta$ has been tuned according to Proposition 19. One can see on the right graph that the global minimizer is preserved and this will be true for any value of $d \in \mathbb{R}$ since (P1) holds for such values of $\theta$. However, on these examples, a local minimizer of $\mathrm{G}_{\text {cap }}$ which is not a minimizer for $\mathrm{G}_{\ell_{0}}$ exists when $\lambda \theta=\sqrt{2 \lambda} a$. This local minimizer $\hat{u} \in(0,1 / \theta)$ verifies

$$
\phi_{\text {cap }}^{\prime}(\theta, \lambda ; \hat{u})=a d-a^{2} \hat{u} \Longleftrightarrow \lambda \theta=a d-a^{2} \hat{u} \Longleftrightarrow \hat{u}=\frac{a d-\lambda \theta}{a^{2}} .
$$

In fact, for any $u_{0} \in(0,1 / \theta)$, there exists $d_{0}=\left(\lambda \theta+a^{2} u_{0}\right) / a \in \mathbb{R}$ for which $u_{0} \neq d_{0} / a$ and is a local minimizer of the associated relaxation $\mathrm{G}_{\text {cap }}$ (using (68)) but is not a minimizer for $\mathrm{G}_{\ell_{0}}$ (i.e. it is different from 0 and $\left.d_{0} / a\right)$. This illustrates the fact that (P2) cannot be verified with the Capped- $\ell_{1}$ penalty.
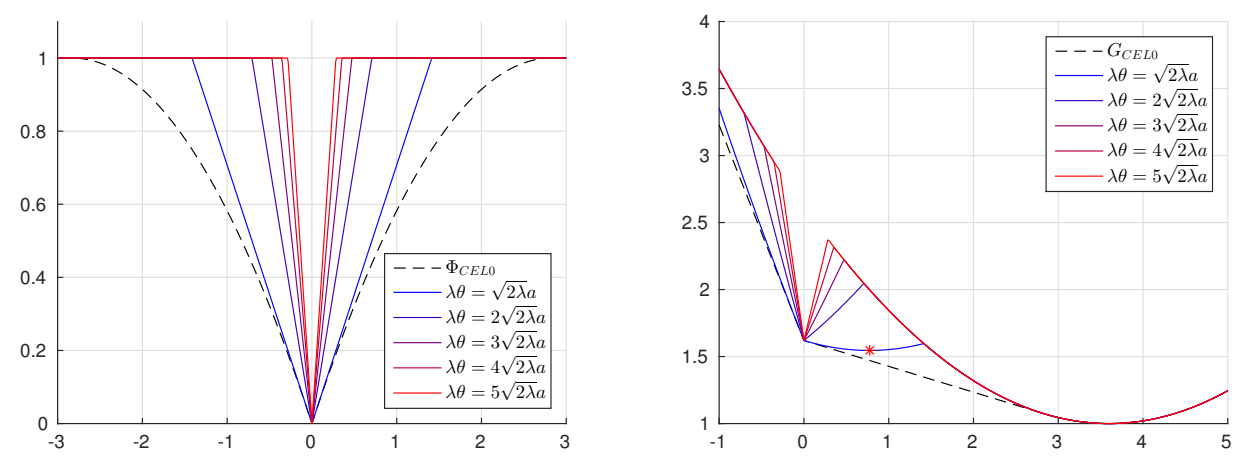

Figure 4: Examples of Capped- $\ell_{1}$ penalties (left) and their associated continuous relaxations $\mathrm{G}_{\text {cap }}$ (right) for which (P1) holds for $a=0.5, \lambda=1$ and $d=1.8$. The red star represents a local minimizer of $\mathrm{G}_{\text {cap }}$ which is not a minimizer for $\mathrm{G}_{\ell_{0}}$.

Similar results for the Capped- $\ell_{1}$ penalty have been shown in $[16,17]$.

\subsection{Smoothly clipped absolute deviation (SCAD)}

The SCAD penalty, which can be seen as a smoothed version of the Capped- $\ell_{1}$, has been proposed by Fan and Li [11] and reads as follows:

$$
\Phi_{\mathrm{SCAD}}(\mathrm{x}):=\sum_{i \in \mathbb{I}_{N}} \phi_{\mathrm{SCAD}}\left(\gamma_{i}, \tilde{\lambda}_{i} ; \mathrm{x}_{i}\right)
$$

where $\gamma_{i} \in(2,+\infty), \tilde{\lambda}_{i} \in \mathbb{R}_{+}^{\star}$ for all $i \in \mathbb{I}_{N}$ and $\phi_{\mathrm{SCAD}}$ is given by: $\forall u \in \mathbb{R}$,

$$
\phi_{\mathrm{SCAD}}(\gamma, \tilde{\lambda} ; u):=
$$

Then one can get a similar result as the one given in Proposition 19 for the Capped- $\ell_{1}$ penalty.

Proposition 20. Let $\left\|a_{i}\right\|<1 / \sqrt{3}$ for all $i \in \mathbb{I}_{N}$. Then, Property (P1) holds for $\mathrm{G}_{\mathrm{SCAD}}$ if and only if

$$
\forall i \in \mathbb{I}_{N}, \frac{\left(\gamma_{i}+1\right) \tilde{\lambda}_{i}^{2}}{2}=\lambda \text { and } 2<\gamma_{i} \leq \frac{1}{\left\|a_{i}\right\|^{2}}-1 .
$$

Moreover, $\mathrm{G}_{\mathrm{SCAD}}$ cannot verify (P2). 
Proof. The proof is given in Appendix B.

Assumption $\left\|a_{i}\right\|<1 / \sqrt{3}\left(i \in \mathbb{I}_{N}\right)$ in Proposition 20 can always be verified by normalizing the columns of $A$ and then multiplying the matrix by a real $\zeta<1 / \sqrt{3}$ since this does not change the problem (it corresponds to a change of variable in $\mathrm{G}_{\ell_{0}}$ ). Figure 5 presents SCAD penalties, with the associated relaxations $\mathrm{G}_{\mathrm{SCAD}}$, verifying the conditions of Proposition 20. The same conclusions as for the Capped- $\ell_{1}$ (Figure 4) can be done.
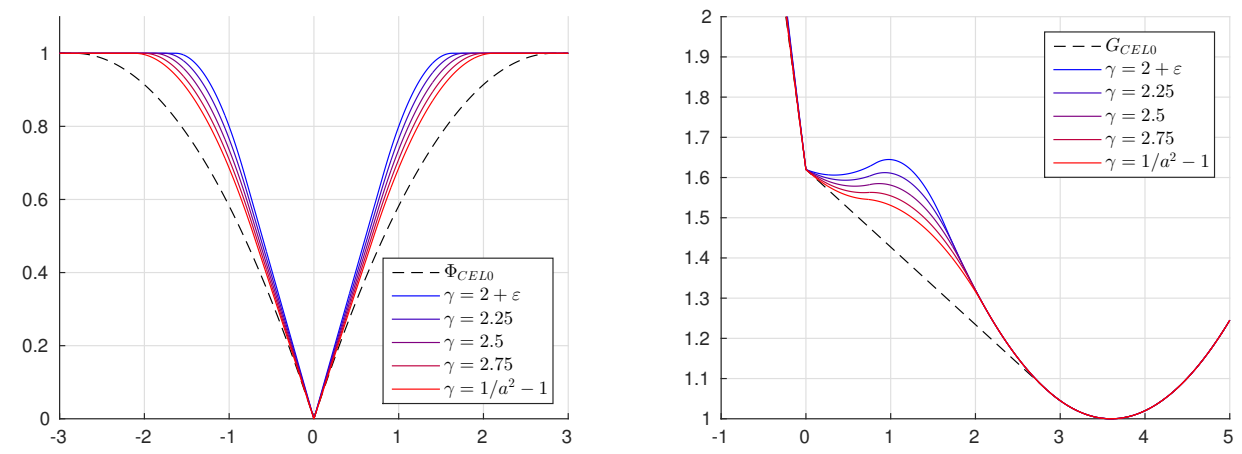

Figure 5: Examples of SCAD penalties (left) and their associated continuous relaxations $\mathrm{G}_{\mathrm{scaD}}$ (right) for which (P1) holds for $a=0.5<1 / \sqrt{3}, \lambda=1$ and $d=1.8$.

\subsection{Minimax concave penalty (MCP)}

The MCP, proposed by Zhang in [31], is defined by

$$
\Phi_{\text {MCP }}(\mathrm{x}):=\sum_{i \in \mathbb{I}_{N}} \phi_{\text {MCP }}\left(\gamma_{i}, \tilde{\lambda}_{i} ; \mathrm{x}_{i}\right),
$$

where $\gamma_{i} \in \mathbb{R}_{+}^{\star}, \tilde{\lambda}_{i} \in \mathbb{R}_{+}^{\star}$ for all $i \in \mathbb{I}_{N}$ and $\phi_{\text {ncp }}$ writes as follows: $\forall u \in \mathbb{R}$,

$$
\begin{aligned}
& \phi_{\text {MCP }}(\gamma, \tilde{\lambda} ; u):= \\
& \quad \tilde{\lambda} \int_{0}^{|u|}(1-x /(\gamma \tilde{\lambda}))_{+} d x=\tilde{\lambda}\left(\frac{\gamma \tilde{\lambda}}{2} \mathbb{1}_{\{|u|>\gamma \tilde{\lambda}\}}+\left(|u|-\frac{u^{2}}{2 \gamma \tilde{\lambda}}\right) \mathbb{1}_{\{|u| \leq \gamma \tilde{\lambda}\}}\right) .
\end{aligned}
$$

From conditions (46) established in the previous section, we can prove that under suitable assumptions on the parameters $\gamma_{i}$ and $\tilde{\lambda}_{i}\left(i \in \mathbb{I}_{N}\right)$, properties (P1) and (P2) hold for the relaxed functional $\mathrm{G}_{\text {мср. }}$. The following proposition states this result.

Proposition 21. Properties (P1) and (P2) hold for $\mathrm{G}_{\text {MсP }}$ if and only if

$$
\forall i \in \mathbb{I}_{N}, \frac{\gamma_{i} \tilde{\lambda}_{i}^{2}}{2}=\lambda \text { and } \gamma_{i}<\frac{1}{\left\|a_{i}\right\|^{2}} .
$$

Proof. By definition of $\Phi_{\text {мср }}$, conditions (46a) and (46d) are verified for all $\gamma_{i}>0$ and $\tilde{\lambda}_{i}>0\left(i \in \mathbb{I}_{N}\right)$. Then, from the results of Section 4, the proof consists in showing that (74) is equivalent to the three 
conditions (46b), (46c) and (46e). From the definition of $\Phi_{\text {мсP }}$, one can easily get that (by symmetry we restrict the proof to $\mathbb{R}_{+}$),

$$
\begin{aligned}
(46 \mathrm{~b}) & \Longleftrightarrow \forall i \in \mathbb{I}_{N}, \frac{\gamma_{i} \tilde{\lambda}_{i}^{2}}{2}=\lambda \text { and } \gamma_{i} \tilde{\lambda}_{i}=\beta^{i+} \leq \frac{\sqrt{2 \lambda}}{\left\|a_{i}\right\|} \\
& \Longleftrightarrow \forall i \in \mathbb{I}_{N}, \frac{\gamma_{i} \tilde{\lambda}_{i}^{2}}{2}=\lambda \text { and } \gamma_{i} \leq \frac{1}{\left\|a_{i}\right\|^{2}} \\
(46 \mathrm{e}) & \Longleftrightarrow \forall i \in \mathbb{I}_{N}, \forall u \in\left(-\gamma_{i} \tilde{\lambda}_{i}, \gamma_{i} \tilde{\lambda}_{i}\right) \backslash\{0\}, \phi_{\text {McP }}^{\prime \prime}(u)=-\frac{1}{\gamma_{i}}<-\left\|a_{i}\right\|^{2} \\
& \Longleftrightarrow \forall i \in \mathbb{I}_{N}, \gamma_{i}<\frac{1}{\left\|a_{i}\right\|^{2}}
\end{aligned}
$$

Clearly, we have that (74) is equivalent to the set of conditions $\{(46 \mathrm{~b}),(46 \mathrm{e})\}$. Hence, it only remains to show that $(74) \Longrightarrow(46 \mathrm{c})$ which is direct from Proposition 12 and completes the proof.

Let $\Phi_{\text {McP }}$ be defined according to the conditions of Proposition 21, then it can be rewritten using only the parameter $\gamma_{i}$ (i.e. removing the dependance on $\tilde{\lambda}_{i}$ ),

$$
\Phi_{\mathrm{MCP}}(\mathrm{x})=\sum_{i \in \mathbb{I}_{N}} \lambda-\frac{1}{2 \gamma_{i}}\left(\left|\mathrm{x}_{i}\right|-\sqrt{2 \lambda \gamma_{i}}\right)^{2} \mathbb{1}_{\left\{\left|\mathrm{x}_{i}\right| \leq \sqrt{2 \lambda \gamma_{i}}\right\}} .
$$

Then, $\forall i \in \mathbb{I}_{N}, \forall \gamma_{i}<\frac{1}{\left\|a_{i}\right\|^{2}}$, the associated functional $\mathrm{G}_{\text {мсP }}$ verifies (P1) and (P2). This defines a sub-family of MCP which are exact continuous approximations of the $\ell_{0}$-norm. Finally, it is worth noting that the inferior limit of this sub-family of MCP is given by

$$
\forall \mathrm{x} \in \mathbb{R}^{N}, \lim _{\substack{\gamma_{i} \rightarrow 1 /\left\|a_{i}\right\|^{2} \\ \forall i \in \mathbb{I}_{N}}} \Phi_{\text {MCP }}(\mathrm{x})=\Phi_{\text {CELo }}(\mathrm{x}) .
$$

Examples of MCP defined following Proposition 21 are shown on Figure 6. This illustrates the fact that, under conditions (74), the MCP is an exact $\ell_{0}$ penalty ensuring to have both (P1) and (P2).
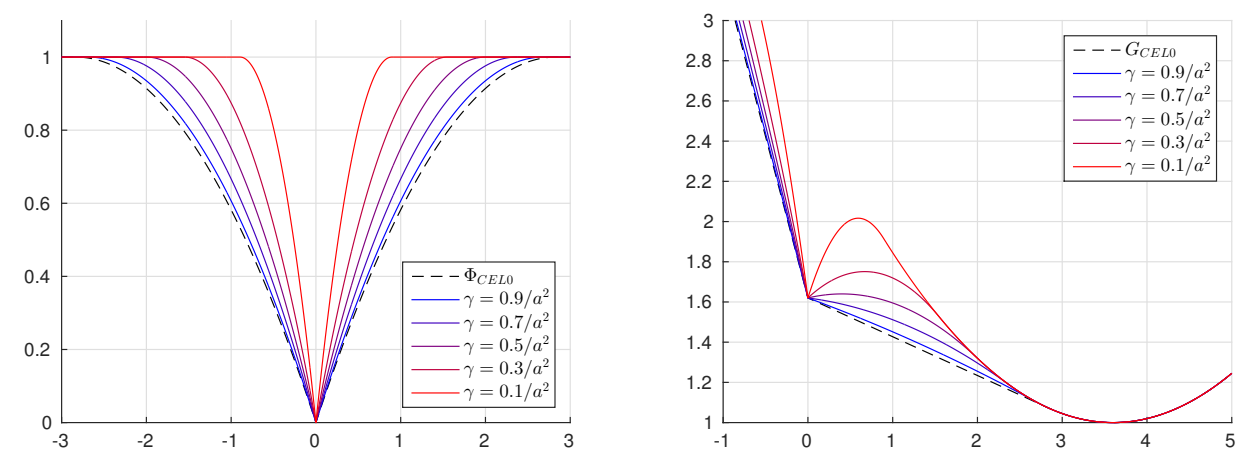

Figure 6: Examples of MCP (left) and their associated continuous relaxations $\mathrm{G}_{\mathrm{MCP}}$ (right) for which (P1) and (P2) hold for $a=0.5, \lambda=1$ and $d=1.8$.

\section{$\mathbf{5 . 4}$ Truncated- $\ell_{p}(0<p<1)$}

It is an evidence that approximations of the $\ell_{0}$-norm such as the $\log$-sum penalty or the $\ell_{p}$-norms $(0<p<1)$ cannot lead to (P1) neither (P2) since they do not verify (46b). However, one can define 
truncated versions of such penalties in the same fashion as the Capped- $\ell_{1}$ studied in Section 5.1. In the following, we analyse the Truncated $-\ell_{p}$ penalties defined by:

$$
\Phi_{\mathrm{TLp}}(\mathrm{x}):=\sum_{i \in \mathbb{I}_{N}} \lambda \min \left\{\left(\theta_{i}\left|\mathrm{x}_{i}\right|+\varepsilon_{i}\right)^{p_{i}}, 1+\varepsilon_{i}^{p_{i}}\right\}-\lambda \varepsilon_{i}^{p_{i}},
$$

where $\theta_{i} \in \mathbb{R}_{+}^{\star}, \varepsilon_{i} \in \mathbb{R}_{+}^{\star}$ and $p_{i} \in(0,1)$ for all $i \in \mathbb{I}_{N}$. Note that the parameters $\varepsilon_{i}$ make the penalty locally Lipschitz at 0 and then fulfil the requirements of Assumption 2.

As for the MCP, given $p_{i}$ and $\varepsilon_{i}\left(i \in \mathbb{I}_{N}\right)$, there exist parameters $\theta_{i}$ for which the continuous relaxation $\mathrm{G}_{\mathrm{TLP}}$ verifies properties $(\mathrm{P} 1)$ and $(\mathrm{P} 2)$.

Proposition 22. Properties (P1) and (P2) hold for $\mathrm{G}_{\mathrm{TLP}}$ if and only if

$$
\forall i \in \mathbb{I}_{N}, \theta_{i} \geq \theta_{0}^{i}:=\left\|a_{i}\right\| \max \left\{\frac{\left(1+\varepsilon_{i}^{p_{i}}\right)^{1 / p_{i}-0.5}}{\sqrt{p_{i}\left(1-p_{i}\right) \lambda}}, \frac{\left(1+\varepsilon_{i}^{p_{i}}\right)^{1 / p_{i}}-\varepsilon_{i}}{\sqrt{2 \lambda}}\right\} .
$$

Proof. Clearly, conditions (46a) and (46d) are verified by definition of $\Phi_{\text {TLp }}$ for all $\theta_{i}>0, p_{i} \in(0,1)$ and $\varepsilon_{i} \in \mathbb{R}_{+}^{*}\left(i \in \mathbb{I}_{N}\right)$. Then, (46e) is equivalent to (by symmetry we restrict the proof to $\mathbb{R}_{+}$): $\forall i \in \mathbb{I}_{N}, \forall u \in\left(0, \frac{1}{\theta_{i}}\left(\left(1+\varepsilon_{i}^{p_{i}}\right)^{1 / p_{i}}-\varepsilon_{i}\right)\right)$,

$$
\left\{\begin{array}{l}
\phi_{\text {TLp }}^{\prime \prime}\left(\theta_{i}, p_{i}, \varepsilon_{i}, \lambda ; u\right)=p_{i}\left(p_{i}-1\right) \lambda \theta_{i}^{2}\left(\theta_{i} u+\varepsilon_{i}\right)^{p_{i}-2} \leq-\left\|a_{i}\right\|^{2} \text { and } \\
\forall \epsilon>0, \exists v_{\epsilon} \in(u-\epsilon, u+\epsilon) \text { s.t. } \phi_{\text {TLP }}^{\prime \prime}\left(\theta_{i}, p_{i}, \varepsilon_{i}, \lambda ; v_{\epsilon}\right)<-\left\|a_{i}\right\|^{2}
\end{array}\right.
$$

where $\phi_{\text {TLP }}(\theta, p, \varepsilon, \lambda ; u):=\lambda \min \left\{(\theta|u|+\varepsilon)^{p}, 1+\varepsilon^{p}\right\}-\lambda \varepsilon^{p}$ for $u \in \mathbb{R}, \theta \in \mathbb{R}_{+}^{\star}, p \in(0,1), \varepsilon \in \mathbb{R}_{+}^{\star}$ and $\lambda \in \mathbb{R}_{+}^{*}$. Since $\phi_{\text {TLp }}^{\prime \prime}\left(\theta_{i}, p_{i}, \varepsilon_{i}, \lambda ; \cdot\right)$ is strictly increasing, (83) reduces to

$$
\begin{gathered}
\forall i \in \mathbb{I}_{N}, \phi_{\text {TLp }}^{\prime \prime}\left(\theta_{i}, p_{i}, \varepsilon_{i}, \lambda ; \beta^{i+}\right)=p_{i}\left(p_{i}-1\right) \lambda \theta_{i}^{2}\left(1+\varepsilon_{i}^{p_{i}}\right)^{1-2 / p_{i}} \leq-\left\|a_{i}\right\|^{2}, \\
\Longleftrightarrow \theta_{i} \geq \frac{\left\|a_{i}\right\|}{\sqrt{p_{i}\left(1-p_{i}\right) \lambda}}\left(1+\varepsilon_{i}^{p_{i}}\right)^{1 / p_{i}-0.5}
\end{gathered}
$$

where $\beta^{i+}=\frac{1}{\theta_{i}}\left(\left(1+\varepsilon_{i}^{p_{i}}\right)^{1 / p_{i}}-\varepsilon_{i}\right)$. Then, one can also see that

$$
\begin{aligned}
(46 \mathrm{~b}) & \Longleftrightarrow \forall i \in \mathbb{I}_{N}, \beta^{i+}=\frac{1}{\theta_{i}}\left(\left(1+\varepsilon_{i}^{p_{i}}\right)^{1 / p_{i}}-\varepsilon_{i}\right) \leq \frac{\sqrt{2 \lambda}}{\left\|a_{i}\right\|}, \\
& \Longleftrightarrow \theta_{i} \geq \frac{\left\|a_{i}\right\|}{\sqrt{2 \lambda}}\left(\left(1+\varepsilon_{i}^{p_{i}}\right)^{1 / p_{i}}-\varepsilon_{i}\right) .
\end{aligned}
$$

Hence $(82) \Longleftrightarrow\{(46 \mathrm{~b}),(46 \mathrm{e})\}$. Finally the foregoing together with Proposition 12 shows that $(82) \Longrightarrow$ (46c) and completes the proof.

A similar analysis could be done for a truncated version of the log-sum penalty or any penalty which is not constant for large values of $|u|$. Finally, Figure 7 shows Truncated- $\ell_{p}$ penalties for which the associated continuous relaxation $\mathrm{G}_{\mathrm{TL}}$ verifies $(\mathrm{P} 1)$ and (P2).

\section{Conclusion}

The variety of " $\ell_{0}$-like" continuous penalties proposed in the literature motivates the analysis of the consistency between the minimizers of the initial and relaxed functionals. Following this idea, we proposed a unified view of exact continuous relaxations for the $\ell_{0}$-regularized least-squares minimization 

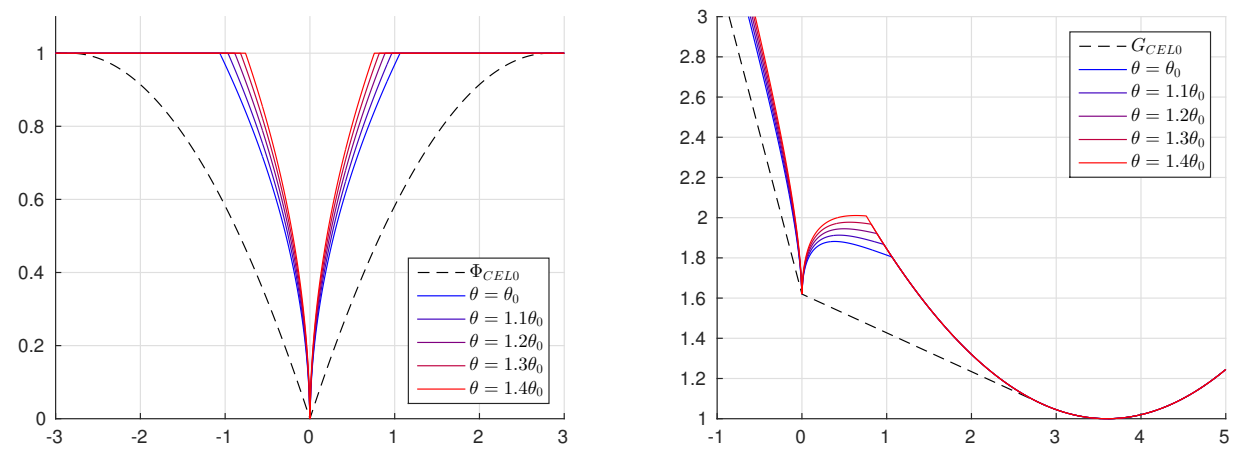

Figure 7: Examples of Truncated- $\ell_{p}$ penalties (left) and their associated continuous relaxations $\mathrm{G}_{\text {TLP }}$ (right) for which (P1) and (P2) hold for $a=0.5, \lambda=1$ and $d=1.8$.

problem. More precisely, we established five necessary and sufficient conditions on the continuous penalty approximating the $\ell_{0}$-norm, such that the resulting continuous objective functional $\tilde{\mathrm{G}}$ preserves all the global minimizers of the initial one $\mathrm{G}_{\ell_{0}}$ (Theorem 13), and that (local) minimizers of $\tilde{\mathrm{G}}$ are also minimizers for $\mathrm{G}_{\ell_{0}}$ (Theorem 18). Although the resulting minimization problem is still nonconvex, an interesting point is that some local minimizers of the initial functional can be removed by the relaxation (Remark 17). Moreover, one can take benefit from the current advances in nonsmooth nonconvex optimization $[1,14,15,22]$ to deal with such an equivalent continuous reformulation. It would be also of interest to consider regularization path strategies as the ones developed in [31, 2] for the MCP in order to obtain a " $\ell_{0}$-regularization path" as done by the recently proposed greedy algorithms in [28].

Finally, the present paper offers a new way to compare continuous penalties approximating the $\ell_{0}$-norm for $\mathrm{G}_{\ell_{0}}$. We showed that, for different penalties proposed over the past, a proper choice of the parameters leads to an exact continuous relaxation of $\mathrm{G}_{\ell_{0}}$ verifying properties (P1) and (P2) (for MCP and the proposed Truncated $-\ell_{p}$ ) while one can only ensure (P1) for other penalties as Capped- $\ell_{1}$ or SCAD. However, in the light of Remark 17, the CEL0 penalty, which is the inferior limit of the derived class of exact penalties, is convex with respect to each variable of the $\mathbb{R}^{N}$ basis and leads to the convex hull in the case where $A$ has nonzero orthogonal columns, seems to be the best choice to continuously approach the $\ell_{0}$-norm in problem (1).

\section{A Proof of Theorem 9}

Since $\mathrm{g}_{0}$ has always two (local) minimizers $\left(u_{1}=0\right.$ and $\left.u_{2}=\frac{d}{a}\right)$ which coincide when $d=0$, one can see that verifying $(\mathrm{P} 2)$ is equivalent to verify

$$
\forall d \in \mathbb{R}, u \in \mathcal{C}_{d} \backslash\left\{0, \frac{d}{a}\right\} \Longrightarrow u \text { is not a (local) minimizer of } \tilde{\mathrm{g}},
$$

where $\mathcal{C}_{d}=\{u \in \mathbb{R}: 0 \in \partial \tilde{\mathrm{g}}(u)\}$ is the set of Clarke critical points of $\tilde{\mathrm{g}}$. Hence the proof consists now in showing that, under (P1), (88) is equivalent to (42).

Let first show the following Lemmas:

Lemma 23. Let $f \in C^{2}(I)$ where $I \subset \mathbb{R}$ and $u \in I$ such that $f^{\prime}(u)=0$. If there exists $\varepsilon_{0}>0$ s.t. $f^{\prime \prime}(v) \geq 0$ for all $v \in\left(u-\varepsilon_{0}, u+\varepsilon_{0}\right)$, then $u$ is a local minimizer of $f$. 
Proof. Using the Taylor formula, for all $v \in\left(u-\varepsilon_{0}, u\right)$ (resp. $\left.v \in\left(u, u+\varepsilon_{0}\right)\right), \exists c \in(v, u)(\operatorname{resp} .(u, v))$ such that

$$
f(v)-f(u)=(v-u) f^{\prime}(u)+\frac{(v-u)^{2}}{2} f^{\prime \prime}(c)
$$

and as $f^{\prime}(u)=0$ and $f^{\prime \prime}(c) \geq 0$ we get

$$
f(v)-f(u) \geq 0 \forall v \in\left(u-\varepsilon_{0}, u+\varepsilon_{0}\right)
$$

so $u$ is a local minimizer of $f$.

Lemma 24. Let $f \in C^{2}(I)$ where $I \subset \mathbb{R}$ and $u \in I$ such that $f^{\prime}(u)=0$. If $f^{\prime \prime}(u)>0$, then $u$ is a local minimizer of $f$.

Proof. The proof is straightforward since the condition $f^{\prime \prime}(u)>0$ implies there exists $\varepsilon_{0}>0$ s.t. for all $v \in\left(u-\varepsilon_{0}, u+\varepsilon_{0}\right)$ we have, by continuity, $f^{\prime \prime}(v)>0$ and the proof follows from Lemma 23.

Then by taking the contrapositive of both Lemmas 23 and 24 we have

Corollary 25. Let $f \in C^{2}(I)$ where $I \subset \mathbb{R}$ and $u \in I$ such that $f^{\prime}(u)=0$, then

$$
\begin{aligned}
& u \text { is } \underline{\text { not a local }} \text { minimizer of } f
\end{aligned} \Longrightarrow\left\{\begin{array}{l}
f^{\prime \prime}(u) \leq 0 \\
\forall \varepsilon>0, \exists v_{\varepsilon} \in(u-\varepsilon, u+\varepsilon) \text { s.t. } f^{\prime \prime}\left(v_{\varepsilon}\right)<0
\end{array}\right.
$$

Lemma 26. Let $f:\left(u-\varepsilon_{0}, u+\varepsilon_{0}\right) \longrightarrow \mathbb{R}$, where $u \in \mathbb{R}$ and $\varepsilon_{0}>0$, be $C^{1}$ on both $\left(u-\varepsilon_{0}, u\right)$ and $\left(u, u+\varepsilon_{0}\right)$ and be continuous at point $u$. We also assume that the limits

$$
\lim _{\substack{v \rightarrow u \\ v<u}} f^{\prime}(v) \text { and } \lim _{\substack{v \rightarrow u \\ v>u}} f^{\prime}(v)
$$

exist and are finite. Then, the two following assertions are true

$$
\begin{aligned}
& \lim _{\substack{v \rightarrow u \\
v<u}} f^{\prime}(v)<0 \text { and } \lim _{\substack{v \rightarrow u \\
v>u}} f^{\prime}(v)>0 \Longrightarrow u \text { is a local minimizer of } f \\
& \lim _{\substack{v \rightarrow u \\
v<u}} f^{\prime}(v)>0 \text { or } \lim _{\substack{v \rightarrow u \\
v>u}} f^{\prime}(v)<0 \Longrightarrow u \text { is } \underline{\text { not a local minimizer of } f}
\end{aligned}
$$

Proof. By definition of limit and by continuity of $f^{\prime}$ on $\left(u-\varepsilon_{0}, u\right)$ and $\left(u, u+\varepsilon_{0}\right)$, there exists $\eta \in\left(0, \varepsilon_{0}\right)$ such that

$$
\forall v \in(u-\eta, u) f^{\prime}(v)<0 \text { and } \forall v \in(u, u+\eta) f^{\prime}(v)>0 .
$$

Hence $f$ is strictly decreasing on $(u-\eta, u)$ and strictly increasing on $(u, u+\eta)$ which completes the proof of (92). Using the same arguments for (93), we show that $f$ is strictly increasing on $(u-\eta, u)$ or strictly decreasing on $(u, u+\eta)$ implying that $u$ is not a local minimizer of $f$ on at least one of these two intervals which completes the proof.

Once again, by taking the contrapositive of (92) in Lemma 26 we have

Corollary 27. Let $f$ be as in Lemma 26, then

$$
u \text { is } \underline{\text { not }} \text { a local minimizer } \Longrightarrow \lim _{\substack{v \rightarrow u \\ v<u}} f^{\prime}(v) \geq 0 \text { or } \lim _{\substack{v \rightarrow u \\ v>u}} f^{\prime}(v) \leq 0 \text {. }
$$


- Proof of $(88) \Longrightarrow(42)$

We split the proof into the two cases $u \in \mathbb{R} \backslash B$ and $u \in B$.

First case: $u \in \mathbb{R} \backslash B$.

Since $B$ contains a finite number of points (Assumption 1), there exists $\varepsilon_{0}>0$ such that $(u-$ $\left.\varepsilon_{0}, u+\varepsilon_{0}\right) \subset \mathbb{R} \backslash B$, and $\tilde{\mathrm{g}}$ is twice differentiable on this interval for all $d$. Hence, from Corollary 25 we get that (88) implies: $\forall d \in \mathbb{R}$,

$$
u \in \mathcal{C}_{d} \backslash\left\{B \cup\left\{\frac{d}{a}\right\}\right\} \Longrightarrow\left\{\begin{array}{l}
\tilde{\mathrm{g}}^{\prime \prime}(u) \leq 0 \\
\forall \varepsilon>0, \exists v_{\varepsilon} \in(u-\varepsilon, u+\varepsilon) \text { s.t. } \tilde{\mathrm{g}}^{\prime \prime}\left(v_{\varepsilon}\right)<0
\end{array}\right.
$$

Now let us take $u \in[-\sqrt{2 \lambda} / a, \sqrt{2 \lambda} / a] \backslash B$ with $\phi^{\prime}(u) \neq 0$. Then, there exists $d_{0} \in \mathbb{R}$ verifying $d_{0} \neq a u$ and

$$
a^{2} u-a d_{0}+\phi^{\prime}(u)=0
$$

which implies, from (16), that $u \in \mathcal{C}_{d_{0}} \backslash\left\{B \cup\left\{\frac{d_{0}}{a}\right\}\right\}$. Thus, it follows from (96) that for all $u \in$ $[-\sqrt{2 \lambda} / a, \sqrt{2 \lambda} / a] \backslash B$ such that $\phi^{\prime}(u) \neq 0$ the right hand side of (96) is a necessary condition for (88). Hence, we have partially shown $(42 \mathrm{~b})$.

Remark 28. Note that, for the moment we have supposed $\phi^{\prime}(u) \neq 0$ and we will get ride of this condition later. We will also prove (42b) for $u \in\left[\beta^{-}, \beta^{+}\right] \backslash B$ rather than $u \in[-\sqrt{2 \lambda} / a, \sqrt{2 \lambda} / a] \backslash B$ later.

\section{Second case: $u \in B$.}

Again, since $B$ contains a finite number of points (Assumption 1), there exists $\varepsilon_{0}>0$ such that $\tilde{g}$ is differentiable on both $\left(u-\varepsilon_{0}, u\right)$ and $\left(u, u+\varepsilon_{0}\right)$. Hence, from Corollary 27 we get that (88) implies: $\forall d \in \mathbb{R}$,

$$
u \in\left(\mathcal{C}_{d} \cap B\right) \backslash\left\{0, \frac{d}{a}\right\} \Longrightarrow \lim _{\substack{v \rightarrow u \\ v<u}} \tilde{\mathrm{g}}^{\prime}(v) \geq 0 \text { or } \lim _{\substack{v \rightarrow u \\ v>u}} \tilde{\mathrm{g}}^{\prime}(v) \leq 0 .
$$

Moreover, since $u$ is a critical point, we get from Proposition 4,

$$
\min \left\{\lim _{\substack{v \rightarrow u \\ v<u}} \phi^{\prime}(v), \lim _{\substack{v \rightarrow u \\ v>u}} \phi^{\prime}(v)\right\} \leq a d-a^{2} u \leq \max \left\{\lim _{\substack{v \rightarrow u \\ v<u}} \phi^{\prime}(v), \lim _{\substack{v \rightarrow u \\ v>u}} \phi^{\prime}(v)\right\},
$$

or equivalently

$$
\min \left\{\lim _{\substack{v \rightarrow u \\ v<u}} \tilde{\mathrm{g}}^{\prime}(v), \lim _{\substack{v \rightarrow u \\ v>u}} \tilde{\mathrm{g}}^{\prime}(v)\right\} \leq 0 \leq \max \left\{\lim _{\substack{v \rightarrow u \\ v<u}} \tilde{\mathrm{g}}^{\prime}(v), \lim _{\substack{v \rightarrow u \\ v>u}} \tilde{\mathrm{g}}^{\prime}(v)\right\}
$$

and the left and right hand side terms of (98) cannot be both equal to zero since $u \in B$ and thus $\tilde{\mathrm{g}}$ is not differentiable at $u$. It follows that left and right derivatives of $\tilde{g}$ at $u$ have different signs. Using this fact, the right hand side of (97) can be rewritten as "(99) or (100) or (101) or (102) or (103)" 
where

$$
\begin{aligned}
& \lim _{\substack{v \rightarrow u \\
v<u}} \tilde{\mathrm{g}}^{\prime}(v)>0 \text { and } \lim _{\substack{v \rightarrow u \\
v>u}} \tilde{\mathrm{g}}^{\prime}(v)<0, \\
& \lim _{\substack{v \rightarrow u \\
v<u}} \tilde{\mathrm{g}}^{\prime}(v)>0 \text { and } \lim _{\substack{v \rightarrow u \\
v>u}} \tilde{\mathrm{g}}^{\prime}(v)=0, \\
& \lim _{\substack{v \rightarrow u \\
v<u}} \tilde{\mathrm{g}}^{\prime}(v)=0 \text { and } \lim _{\substack{v \rightarrow u \\
v>u}} \tilde{\mathrm{g}}^{\prime}(v)<0, \\
& \lim _{\substack{v \rightarrow u \\
v<u}} \tilde{\mathrm{g}}^{\prime}(v)<0 \text { and } \lim _{\substack{v \rightarrow u \\
v>u}} \tilde{\mathrm{g}}^{\prime}(v)=0, \\
& \lim _{\substack{v \rightarrow u \\
v<u}} \tilde{\mathrm{g}}^{\prime}(v)=0 \text { and } \lim _{\substack{v \rightarrow u \\
v>u}} \tilde{\mathrm{g}}^{\prime}(v)>0 .
\end{aligned}
$$

Note that since the quadratic term in $\tilde{g}$ is differentiable on $\mathbb{R}$, we have

$$
\left.\left.\lim _{\substack{v \rightarrow u \\ v>u}} \tilde{\mathrm{g}}^{\prime}(v) \text { (resp. } \lim _{\substack{v \rightarrow u \\ v<u}} \tilde{\mathrm{g}}^{\prime}(v)\right)=\lim _{\substack{v \rightarrow u \\ v>u}} \phi^{\prime}(v) \text { (resp. } \lim _{\substack{v \rightarrow u \\ v<u}} \phi^{\prime}(v)\right)+a^{2} u-a d \text {. }
$$

This will be used in what follows. Now, assume that there exists $d_{0} \in \mathbb{R}$ such that $u \in\left(\mathcal{C}_{d_{0}} \cap B\right) \backslash\left\{0, \frac{d_{0}}{a}\right\}$ is not a local minimizer of $\tilde{g}_{d_{0}}$ and that (102) holds (one can do the same development for (103)).

Condition (102) means

$$
\lim _{\substack{v \rightarrow u \\ v<u}} \phi^{\prime}(v)<a d_{0}-a^{2} u=\lim _{\substack{v \rightarrow u \\ v>u}} \phi^{\prime}(v)
$$

Then, there exists $d_{1}<d_{0}$ such that $u \neq d_{1} / a$ and

$$
\begin{aligned}
& \lim _{\substack{v \rightarrow u \\
v<u}} \phi^{\prime}(v)<a d_{1}-a^{2} u<a d_{0}-a^{2} u=\lim _{\substack{v \rightarrow u \\
v>u}} \phi^{\prime}(v), \\
\Longleftrightarrow & \lim _{\substack{v \rightarrow u \\
v<u}} \phi^{\prime}(v)+a^{2} u-a d_{1}<0<\lim _{\substack{v \rightarrow u \\
v>u}} \phi^{\prime}(v)+a^{2} u-a d_{1}, \\
\Longleftrightarrow & \lim _{\substack{v \rightarrow u \\
v<u}} \tilde{\mathrm{g}}_{d_{1}}^{\prime}(v)<0<\lim _{\substack{v \rightarrow u \\
v>u}} \tilde{\mathrm{g}}_{d_{1}}^{\prime}(v),
\end{aligned}
$$

and Proposition 4 tell us that $u \in\left(\mathcal{C}_{d_{1}} \cap B\right) \backslash\left\{0, \frac{d_{1}}{a}\right\}$. However, from (107) and (92) of Lemma 26, $u$ is a local minimizer of $\tilde{g}_{d_{1}}$. This violates (88) and the right hand side of (97) is finally reduced to "(99) or (100) or (101)" implying

$$
\lim _{\substack{v \rightarrow u \\ v<u}} \tilde{\mathrm{g}}^{\prime}(v)>\lim _{\substack{v \rightarrow u \\ v>u}} \tilde{\mathrm{g}}^{\prime}(v) \Longleftrightarrow \lim _{\substack{v \rightarrow u \\ v<u}} \phi^{\prime}(v)>\lim _{\substack{v \rightarrow u \\ v>u}} \phi^{\prime}(v) .
$$

Finally, as for the first case, one can see that for all $u \in B \backslash\{0\}$, there exists $d_{0} \in \mathbb{R}$, such that $u \neq d_{0} / a$ and

$$
a d_{0}-a^{2} u \in\left[\bar{\delta}^{u}, \underline{\delta}^{u}\right]
$$

which implies, from $(16)$, that $u \in\left(\mathcal{C}_{d_{0}} \cap B\right) \backslash\left\{0, \frac{d_{0}}{a}\right\}$.

Hence, from the above, we have proved that

$$
u \in B \backslash\{0\} \Longrightarrow \lim _{\substack{v \rightarrow u \\ v<u}} \phi^{\prime}(v)>\lim _{\substack{v \rightarrow u \\ v>u}} \phi^{\prime}(v)
$$

is a necessary condition for (88). 
To summarize, from the two cases $u \notin B$ and $u \in B$ we got the following necessary conditions for $(88)$

$$
\begin{aligned}
\forall u \in & {[-\sqrt{2 \lambda} / a, \sqrt{2 \lambda} / a] \backslash\left\{\{0\} \cup\left\{u \notin B: \phi^{\prime}(u)=0\right\}\right\}, } \\
& \begin{cases}\left\{\begin{array}{l}
\phi^{\prime \prime}(u) \leq-a^{2} \text { and } \\
\forall \varepsilon>0, \exists v_{\varepsilon} \in(u-\varepsilon, u+\varepsilon) \text { s.t. } \phi^{\prime \prime}\left(v_{\varepsilon}\right)<-a^{2}
\end{array}\right. & \text { if } u \notin B, \\
\lim _{\substack{v \rightarrow u \\
v<u}} \phi^{\prime}(v)>\lim _{\substack{v \rightarrow u \\
v>u}} \phi^{\prime}(v) & \text { if } u \in B,\end{cases}
\end{aligned}
$$

Conclusion (109) is not exactly the one stated in Theorem 9: we have to get ride of the assumption $\phi^{\prime}(u) \neq 0$ and we have to define $\beta^{-}$and $\beta^{+}$. We get from (40)

$$
\lim _{\substack{u \rightarrow 0 \\ u>0}} \phi^{\prime}(u) \geq \lim _{\substack{u \rightarrow 0 \\ u>0}} \phi_{\text {CELO }}^{\prime}(u)=\sqrt{2 \lambda} a>0 \text { and } \lim _{\substack{u \rightarrow \sqrt{2 \lambda} / a \\ u>\sqrt{2 \lambda} / a}} \phi^{\prime}(u)=0 .
$$

Moreover, from (109), we get that the "derivative" of $\phi$ is a discontinuous (at points in $B$ ) decreasing function on $[0, \sqrt{2 \lambda} / a]$. Hence, if there is $\bar{u} \in[0, \sqrt{2 \lambda} / a] \backslash B$ such that $\phi^{\prime}(\bar{u})=0$, then $\phi^{\prime}(u)=0$ $\forall u \in[\bar{u},+\infty)$. A similar reasoning can be done on $\mathbb{R}_{-}$and then, defining $\beta^{-}$and $\beta^{+}$as in the statement of the theorem, we get that $B \subset\left[\beta^{-}, \beta^{+}\right]$and that $\forall u \in\left(\beta^{-}, \beta^{+}\right) \backslash B, \phi^{\prime}(u) \neq 0$. Thus, (109) becomes:

$$
\begin{aligned}
& \forall u \in B \backslash\{0\}, \lim _{\substack{v \rightarrow u \\
v<u}} \phi^{\prime}(v)>\lim _{\substack{v \rightarrow u \\
v>u}} \phi^{\prime}(v) \\
& \forall u \in\left(\beta^{-}, \beta^{+}\right) \backslash B,\left\{\begin{array}{l}
\phi^{\prime \prime}(u) \leq-a^{2} \text { and } \\
\forall \varepsilon>0, \exists v_{\varepsilon} \in(u-\varepsilon, u+\varepsilon) \text { s.t. } \phi^{\prime \prime}\left(v_{\varepsilon}\right)<-a^{2}
\end{array}\right.
\end{aligned}
$$

which completes the proof of $\Longleftarrow$.

- Proof of $(42) \Longrightarrow(88)$

Let $u \in \mathcal{C}_{d} \backslash\left\{0, \frac{d}{a}\right\}$ for $d \in \mathbb{R}$. Then, from the critical point characterization (16) and the definition of the bounds $\beta^{-}$and $\beta^{+}$, one gets that $u \in\left[\beta^{-}, \beta^{+}\right] \backslash\{0\}$. Indeed a critical point of $\tilde{g}$ on the constant part of $\phi$ necessarily verifies $u=d / a$ from (16). Moreover, if $\beta^{-} \notin B$ (resp. $\beta^{+} \notin B$ ) then $u \neq \beta^{-}$ (resp. $u \neq \beta^{+}$) otherwise we will have $\phi^{\prime}(u)=0$ and thus $u=d / a$ which is not compatible with the above. Then, from (42) we distinguish two cases:

- if $u \in B$, then (42a) together with the differentiability of the quadratic term in $\tilde{g}$ implies that

$$
\lim _{\substack{v \rightarrow u \\ v<u}} \tilde{\mathrm{g}}^{\prime}(v)=a^{2} u-a d+\lim _{\substack{v \rightarrow u \\ v<u}} \phi^{\prime}(v) \underset{(42 \mathrm{a})}{>} a^{2} u-a d+\lim _{\substack{v \rightarrow u \\ v>u}} \phi^{\prime}(v)=\lim _{\substack{v \rightarrow u \\ v>u}} \tilde{\mathrm{g}}^{\prime}(v),
$$

which, combined with the fact that the left and right derivatives of $\tilde{g}$ at the critical point $u$ have different signs, implies that we can only have "(99) or (100) or (101)". Then the conclusion is straightforward from point (93) in Lemma 26.

- if $u \notin B$, then (42b) together with Assumption 1 imply that $\exists \varepsilon_{0}$ such that $\tilde{\mathrm{g}}$ is concave on $\left(u-\varepsilon_{0}, u+\varepsilon_{0}\right)$. Moreover we have using Taylor formula

$$
\forall v \in\left(u-\varepsilon_{0}, u+\varepsilon_{0}\right), \quad \tilde{\mathrm{g}}(v)-\tilde{\mathrm{g}}(u)=\frac{(v-u)^{2}}{2} \tilde{\mathrm{g}}^{\prime \prime}(c),
$$

where $c \in(v, u)$ if $v<u$, or $c \in(u, v)$ if $u<v$. Then we have $\tilde{\mathrm{g}}^{\prime \prime}(c) \leq 0$ and $\tilde{\mathrm{g}}(v) \leq \tilde{\mathrm{g}}(u)$, $\forall v \in\left(u-\varepsilon_{0}, u+\varepsilon_{0}\right)$. But $\exists v_{\varepsilon_{0}} \in\left(u-\varepsilon_{0}, u+\varepsilon_{0}\right)$ such that $\tilde{\mathrm{g}}\left(v_{\varepsilon_{0}}\right)<\tilde{\mathrm{g}}(u)$ otherwise we would have 
$\tilde{\mathrm{g}}(v)=\tilde{\mathrm{g}}(u)=c s t, \forall v \in\left(u-\varepsilon_{0}, u+\varepsilon_{0}\right)$, which would imply that $\tilde{\mathrm{g}}$ is constant on $\left(u-\varepsilon_{0}, u+\varepsilon_{0}\right)$, implying $\tilde{\mathrm{g}}^{\prime \prime}(v)=0, \forall v \in\left(u-\varepsilon_{0}, u+\varepsilon_{0}\right)$.

This is impossible from $(42 \mathrm{~b})$ because

$$
\forall \varepsilon>0, \exists v_{\varepsilon} \in(u-\varepsilon, u+\varepsilon) \text { s.t. } \phi^{\prime \prime}\left(v_{\varepsilon}\right)<-a^{2} \Leftrightarrow \tilde{\mathrm{g}}^{\prime \prime}\left(v_{\varepsilon}\right)<0 .
$$

Hence, for all $\varepsilon>0, \exists v_{\varepsilon} \in(u-\varepsilon, u+\varepsilon)$ such that $\tilde{\mathrm{g}}\left(v_{\varepsilon}\right)<\tilde{\mathrm{g}}(u)$ showing that $u$ is not a local minimizer of $\tilde{g}$.

\section{B Proof of Proposition 20}

By definition of $\Phi_{\text {scAD }}$, condition (46a) holds for all $\gamma_{i}>2$ and $\tilde{\lambda}_{i}>0\left(i \in \mathbb{I}_{N}\right)$. Moreover, one can easily get

$$
\begin{aligned}
& (46 \mathrm{~b}) \Longleftrightarrow \forall i \in \mathbb{I}_{N}, \frac{\left(\gamma_{i}+1\right) \tilde{\lambda}_{i}^{2}}{2}=\lambda \text { and } \gamma_{i} \tilde{\lambda}_{i}=\beta^{i+} \leq \frac{\sqrt{2 \lambda}}{\left\|a_{i}\right\|}, \\
& (46 \mathrm{c}) \Longrightarrow \forall i \in \mathbb{I}_{N}, \tilde{\lambda}_{i} \geq \sqrt{2 \lambda}\left\|a_{i}\right\|,
\end{aligned}
$$

where the last implication comes from the same argument as used in the proof of Proposition 19 (note that by symmetry $\left.\beta^{i-}=-\beta^{i+}\right)$. Let us now verify if the above conditions on $\gamma_{i}$ and $\tilde{\lambda}_{i}$ can hold simultaneously. We have, $\forall i \in \mathbb{I}_{N}$

$$
\begin{aligned}
& \tilde{\lambda}_{i}=\sqrt{\frac{2 \lambda}{\gamma_{i}+1}} \text { and } \frac{\gamma_{i}}{\sqrt{\gamma_{i}+1}} \leq \frac{1}{\left\|a_{i}\right\|} \text { and } \frac{1}{\sqrt{\gamma_{i}+1}} \geq\left\|a_{i}\right\|, \\
& \underset{\gamma_{i}>2}{\Longleftrightarrow} \tilde{\lambda}_{i}=\sqrt{\frac{2 \lambda}{\gamma_{i}+1}} \text { and }\left\|a_{i}\right\| \leq \frac{1}{\sqrt{\gamma_{i}+1}}<\frac{\sqrt{\gamma_{i}+1}}{\gamma_{i}} \text { and }\left\|a_{i}\right\|<\frac{1}{\sqrt{3}} \text {. } \\
& \Longleftrightarrow \tilde{\lambda}_{i}=\sqrt{\frac{2 \lambda}{\gamma_{i}+1}} \text { and } \gamma_{i} \leq \frac{1}{\left\|a_{i}\right\|^{2}}-1 \text { and }\left\|a_{i}\right\|<\frac{1}{\sqrt{3}} \text {. }
\end{aligned}
$$

To conclude the proof, we need to show that $(71) \Longrightarrow(46 \mathrm{c})$. By symmetry, we restrict the proof to $\mathbb{R}_{+}$. Clearly, using the same arguments as in the proof of Proposition 19, we have under (71)

$$
\forall i \in \mathbb{I}_{N}, \forall u \in\left(0, \tilde{\lambda}_{i}\right], \phi_{\text {CELo }}\left(\left\|a_{i}\right\|, \lambda ; u\right)<\phi_{\text {SCAD }}\left(\gamma_{i}, \tilde{\lambda}_{i} ; u\right),
$$

since $(71) \Longrightarrow \tilde{\lambda}_{i} \geq \sqrt{2 \lambda}\left\|a_{i}\right\|$. Then, $\forall i \in \mathbb{I}_{N}, \forall u \in\left[\tilde{\lambda}_{i}, \gamma_{i} \tilde{\lambda}_{i}\right]$, we have

$$
\begin{gathered}
\phi_{\text {CELo }}\left(\left\|a_{i}\right\|, \lambda ; u\right)=P_{1}(u)=-\frac{\left\|a_{i}\right\|^{2}}{2} u^{2}+\sqrt{2 \lambda}\left\|a_{i}\right\| u, \\
\phi_{\mathrm{SCAD}}\left(\gamma_{i}, \tilde{\lambda}_{i} ; u\right)=P_{2}(u)=-\frac{\tilde{\lambda}_{i}^{2}-2 \gamma_{i} \tilde{\lambda}_{i}|u|+u^{2}}{2\left(\gamma_{i}-1\right)}
\end{gathered}
$$

where $\left(P_{1}, P_{2}\right) \in\left(\mathbb{R}^{2}[X]\right)^{2}$ are two order 2 polynomials. Let us consider the order two polynomial $Q=P_{2}-P_{1}$. Then it follows from (114) and (119) that $Q\left(\tilde{\lambda}_{i}\right)>0$ and $Q\left(\gamma_{i} \tilde{\lambda}_{i}\right) \geq 0$ (since (71) $\Longrightarrow$ (114)). Moreover, one can see that

$$
\forall u \in\left[\tilde{\lambda}_{i}, \gamma_{i} \tilde{\lambda}_{i}\right], Q^{\prime \prime}(u)=\left\|a_{i}\right\|^{2}-\frac{1}{\gamma_{i}-1}<0
$$


since $(71) \Rightarrow\left\|a_{i}\right\|^{2} \leq \frac{1}{\gamma_{i}+1} \underset{\left(\gamma_{i}>2\right)}{<} \frac{1}{\gamma_{i}-1}$. Hence $Q$ is strictly concave on $\left[\tilde{\lambda}_{i}, \gamma_{i} \tilde{\lambda}_{i}\right]$ which, with $Q\left(\tilde{\lambda}_{i}\right)>0$ and $Q\left(\gamma_{i} \tilde{\lambda}_{i}\right) \geq 0$, implies that $Q(u)>0 \forall u \in\left[\tilde{\lambda}_{i}, \gamma_{i} \tilde{\lambda}_{i}\right)$. This shows that $(71) \Longrightarrow(46 \mathrm{c})$. Finally, the fact that $\mathrm{G}_{\mathrm{SCAD}}$ cannot verify (P2) follows from similar arguments as the ones used in the proof of Proposition 19.

\section{Acknowledgements}

The authors would like to thanks the referees for the relevant comments and suggestions helpful to improve the manuscript. In particular they are very grateful to the anonymous reviewer 2 .

\section{References}

[1] Hedy Attouch, Jérôme Bolte, and Benar Fux Svaiter. Convergence of descent methods for semialgebraic and tame problems: proximal algorithms, forward-backward splitting, and regularized Gauss-Seidel methods. Mathematical Programming, 137(1-2):91-129, 2013.

[2] Aurélie Boisbunon. Model selection: a decision-theoretic approach. Thesis, Université de Rouen, 2013.

[3] S. Bourguignon, J. Ninin, H. Carfantan, and M. Mongeau. Exact sparse approximation problems via mixed-integer programming: Formulations and computational performance. IEEE Transactions on Signal Processing, 2015.

[4] Leo Breiman. Better subset regression using the nonnegative garrote. Technometrics, 37(4):373$384,1995$.

[5] Emmanuel J Candès, Justin Romberg, and Terence Tao. Robust uncertainty principles: Exact signal reconstruction from highly incomplete frequency information. IEEE Transactions on Information Theory, 52(2):489-509, 2006.

[6] Emmanuel J Candes, Michael B Wakin, and Stephen P Boyd. Enhancing sparsity by reweighted $\ell_{1}$ minimization. Journal of Fourier analysis and applications, 14(5-6):877-905, 2008.

[7] Emilie Chouzenoux, Anna Jezierska, Jean-Christophe Pesquet, and Hugues Talbot. A majorizeminimize subspace approach for $\ell_{2}-\ell_{0}$ image regularization. SIAM Journal on Imaging Sciences, 6(1):563-591, 2013.

[8] Frank H Clarke. Optimization and nonsmooth analysis, volume 5. SIAM, 1990.

[9] Hongbo Dong, Kun Chen, and Jeff Linderoth. Regularization vs. Relaxation: A conic optimization perspective of statistical variable selection. Optimization Online, 2015.

[10] David L Donoho. For most large underdetermined systems of linear equations the minimal $\ell_{1}$ norm solution is also the sparsest solution. Communications on Pure and Applied Mathematics, 59(6):797-829, 2006.

[11] Jianqing Fan and Runze Li. Variable selection via nonconcave penalized likelihood and its oracle properties. Journal of the American Statistical Association, 96(456):1348-1360, 2001.

[12] Simon Foucart and Ming-Jun Lai. Sparsest solutions of underdetermined linear systems via $\ell_{q^{-}}$ minimization for $0<q \leq 1$. Applied and Computational Harmonic Analysis, 26(3):395-407, 2009. 
[13] GM Fung and OL Mangasarian. Equivalence of minimal $\ell_{0^{-}}$and $\ell_{p^{-}}$norm solutions of linear equalities, inequalities and linear programs for sufficiently small p. Journal of optimization theory and applications, 151(1):1-10, 2011.

[14] Gilles Gasso, Alain Rakotomamonjy, and Stéphane Canu. Recovering sparse signals with a certain family of nonconvex penalties and DC programming. IEEE Transactions on Signal Processing, 57(12):4686-4698, 2009.

[15] Pinghua Gong, Changshui Zhang, Zhaosong Lu, Jianhua Huang, and Jieping Ye. A General Iterative Shrinkage and Thresholding Algorithm for Non-convex Regularized Optimization Problems. In Proceedings of The 30th International Conference on Machine Learning, pages 37-45, 2013.

[16] Hoai An Le Thi, T Pham Dinh, Hoai Minh Le, and Xuan Thanh Vo. DC approximation approaches for sparse optimization. European Journal of Operational Research, 244(1):26-46, 2015.

[17] Hoai An Le Thi, Hoai Minh Le, and Tao Pham Dinh. Feature selection in machine learning: an exact penalty approach using a difference of convex function algorithm. Machine Learning, pages $1-24,2014$.

[18] Stéphane G Mallat and Zhifeng Zhang. Matching pursuits with time-frequency dictionaries. IEEE Transactions on Signal Processing, 41(12):3397-3415, 1993.

[19] Balas Kausik Natarajan. Sparse approximate solutions to linear systems. SIAM journal on computing, 24(2):227-234, 1995.

[20] Mila Nikolova. Description of the minimizers of least squares regularized with $\ell_{0}$-norm. Uniqueness of the global minimizer. SIAM Journal on Imaging Sciences, 6(2):904-937, 2013.

[21] Mila Nikolova. Relationship between the optimal solutions of least squares regularized with $\ell_{0^{-}}$ norm and constrained by k-sparsity. Applied and Computational Harmonic Analysis, 41(1):237$265,2016$.

[22] P. Ochs, A. Dosovitskiy, T. Brox, and T. Pock. On Iteratively Reweighted Algorithms for Nonsmooth Nonconvex Optimization in Computer Vision. SIAM Journal on Imaging Sciences, $8(1): 331-372,2015$.

[23] Yagyensh Chandra Pati, Ramin Rezaiifar, and PS Krishnaprasad. Orthogonal matching pursuit: Recursive function approximation with applications to wavelet decomposition. In Signals, Systems and Computers, 1993. 1993 Conference Record of The Twenty-Seventh Asilomar Conference on, pages 40-44. IEEE, 1993.

[24] Mert Pilanci, Martin J Wainwright, and Laurent El Ghaoui. Sparse learning via Boolean relaxations. Mathematical Programming, 151(1):63-87, 2015.

[25] Audrey Repetti, Mai Quyen Pham, Laurent Duval, Emilie Chouzenoux, and Jean-Christophe Pesquet. Euclid in a taxicab: Sparse blind deconvolution with smoothed $\ell_{1} / \ell_{2}$ regularization. IEEE Signal Processing Letters, 22(5):539-543, 2015.

[26] Emmanuel Soubies, Laure Blanc-Féraud, and Gilles Aubert. A Continuous Exact $\ell_{0}$ Penalty (CEL0) for Least Squares Regularized Problem. SIAM Journal on Imaging Sciences, 8(3):16071639, 2015.

[27] Charles Soussen, Jérôme Idier, David Brie, and Junbo Duan. From Bernoulli-Gaussian deconvolution to sparse signal restoration. IEEE Transactions on Signal Processing, 59(10):4572-4584, 2011. 
[28] Charles Soussen, Jérome Idier, Junbo Duan, and David Brie. Homotopy based algorithms for L0-regularized least-squares. IEEE Transactions on Signal Processing, 63(13):3301-3316, 2015.

[29] Joel A Tropp. Greed is good: Algorithmic results for sparse approximation. IEEE Transactions on Information Theory, 50(10):2231-2242, 2004.

[30] Cun-Hui Zhang. Discussion: One-step sparse estimates in nonconcave penalized likelihood models. The Annals of Statistics, 36(4):1553-1560, 2008.

[31] Cun-Hui Zhang. Nearly unbiased variable selection under minimax concave penalty. The Annals of Statistics, pages 894-942, 2010.

[32] Na Zhang and Qia Li. On optimal solutions of the constrained $\ell_{0}$ regularization and its penalty problem. Inverse Problems, 33(2), 2017.

[33] Tong Zhang. Multi-stage convex relaxation for learning with sparse regularization. In Advances in Neural Information Processing Systems, pages 1929-1936, 2009.

[34] Hui Zou. The adaptive lasso and its oracle properties. Journal of the American statistical association, 101(476):1418-1429, 2006. 